\title{
Earth-Viewing L-Band Radiometer Sensing of Sea Surface Scattered Celestial Sky Radiation-Part II: Application to SMOS
}

\author{
Nicolas Reul, Joseph Tenerelli, Nicolas Floury, and Bertrand Chapron
}

\begin{abstract}
5 Abstract-We examine how the rough sea surface scattering 6 of L-band celestial sky radiation might affect the measurements 7 of the future European Space Agency Soil Moisture and Ocean 8 Salinity (SMOS) mission. For this purpose, we combined data 9 from several surveys to build a comprehensive all-sky L-band 10 celestial sky brightness temperature map for the SMOS mission 11 that includes the continuum radiation and the hydrogen line 12 emission rescaled for the SMOS bandwidth. We also constructed a 13 separate map of strong and very localized sources that may exhibit 14 L-band brightness temperatures exceeding $1000 \mathrm{~K}$. Scattering by 15 the roughened ocean surface of radiation from even the strongest 16 localized sources is found to reduce the contributions from these 17 localized strong sources to negligible levels, and rough surface 18 scattering solutions may be obtained with a map much coarser 19 than the original continuum maps. In rough ocean surface condi20 tions, the contribution of the scattered celestial noise to the recon21 structed brightness temperatures is not significantly modified by 22 the synthetic antenna weighting function, which makes integration 23 over the synthetic beam unnecessary. The contamination of the 24 reconstructed brightness temperatures by celestial noise exhibits 25 a strong annual cycle with the largest contamination occurring 26 in the descending swaths in September and October, when the 27 specular projection of the field of view is aligned with the galactic 28 equator. Ocean surface roughness may alter the contamination by 29 over $0.1 \mathrm{~K}$ in $30 \%$ of the SMOS measurements. Given this poten30 tially large impact of surface roughness, an operational method is 31 proposed to account for it in the SMOS level 2 sea surface salinity 32 algorithm.
\end{abstract}

33 Index Terms-Microwave radiometry, sea surface electromag34 netic scattering.

\section{INTRODUCTION}

36

37 ELESTIAL sky L-band radiation scattered by the ocean surface can contaminate spaceborne measurements of up38 welling sea surface brightness temperature used to retrieve sea 39 surface salinity (SSS). The sensitivity of the linearly polarized 40 sea surface brightness temperature to salinity ranges from about 410.2 to $0.8 \mathrm{~K} / \mathrm{psu}$ [1] (depending on ocean surface temperature, 42 incidence angle, and polarization). Since the open ocean surface

Manuscript received March 1, 2007; revised June 20, 2007.

N. Reul, J. Tenerelli, and B. Chapron are with the Laboratoire d'Océanographie Spatiale, Institut Français de Recherche et d'Exploitation de la Mer, 29280 Brest, France (e-mail: nreul@ifremer.fr).

N. Floury is with the Electromagnetics and Space Environments Division, European Space Research and Technology Centre (ESTEC), European Space Agency, 2200 AG Noordwijk, The Netherlands.

Color versions of one or more of the figures in this paper are available online at http://ieeexplore.ieee.org.

Digital Object Identifier 10.1109/TGRS.2007.914804 salinity generally ranges from 32 to 37 psu, the expected 43 dynamical range of L-band emission brightness temperatures 44 associated with variations in SSS alone is small relative to the 45 total brightness temperature, which is less than approximately 46 $4 \mathrm{~K}$ for open ocean conditions.

For the Aquarius/SAC-D mission, it was reported in [2] 48 that, under the assumption of a flat perfectly conducting Earth 49 surface (with a reflectivity of 1 ), the total celestial sky radiation 50 contribution to the antenna temperature varies from a little 51 less than $4 \mathrm{~K}$ to more than $9 \mathrm{~K}$. For a perfectly flat dielectric 52 sea surface, the reflectivity may range from about $30 \%$ to 53 $80 \%$ at $1.4 \mathrm{GHz}$ for incidence angles below $50^{\circ}$, depending 54 on the SSS, sea surface physical temperature, and observation 55 polarization. In this case, the contamination ranges from about 56 1 to $7 \mathrm{~K}$. As discussed in [3] (hereinafter referred to as Part I), 57 ocean surface roughness both decreases the surface reflectivity 58 and directionally spreads the impact of the source brightness. 59 For specular points in the vicinity of the galactic equator, the 60 spreading effect of the rough surface greatly reduces the impact 61 along the equator and broadens the contamination far beyond 62 the narrow bright source concentrated along the equator. Never- 63 theless, for specular points far from strong sources, sea surface 64 roughness has a negligible impact on the reflected signal. 65 Overall, the intensity of the scattered celestial noise ranges from 66 approximately $30 \%$ to $70 \%$ of the flat ocean surface reflected 67 values, with most of the variation associated with the directional 68 spreading of the radiation.

The nonuniform distribution of celestial radiation has an 70 important systematic impact on the measurements. The future 71 European Space Agency Soil Moisture and Ocean Salinity 72 (SMOS) and National Aeronautics and Space Administration/ 73 Comisión Nacional de Actividades Espaciales Aquarius/ 74 SAC-D satellites that are dedicated to SSS remote sensing will 75 be launched in the near future into sun-synchronous orbits, and 76 considering these orbits along with the Earth's orbit around the 77 sun, the celestial sky glitter contamination will exhibit strong 78 geographic and seasonal dependence. As such, flagging and 79 correction strategies for such contamination must be developed 80 to reduce large-scale seasonal and geographical biases in the 81 retrieved surface salinity fields.

To achieve the 0.1-psu accuracy goal for the retrieved salin- 83 ity, the sky glitter contribution must be estimated with an 84 uncertainty not exceeding $0.05 \mathrm{~K}$. This is a stringent constraint 85 that may be difficult to satisfy given the accuracies of both the 86 future SMOS radiometric measurements and the sky brightness 87 
88 temperature maps. This constraint also presents potential dif89 ficulties for the forward modeling of the scene brightness 90 temperatures since this modeling is plagued by uncertainties 91 and potential biases associated with rough sea surface scattering 92 and emissivity models. Moreover, for the SMOS mission, the 93 multidirectional nature of the measurements incorporated into 94 the salinity retrieval at any given point on Earth results in a 95 potentially wide range of celestial noise contamination for any 96 given retrieval, so that failure to correct for the contamination 97 prior to salinity retrieval may result in retrieved salinity errors 98 and biases that are not easily correctable by further processing 99 at a later stage.

100 The focus of this paper is given as follows: 1) to analyze the 101 expected annual cycle of contamination of SMOS multiangular 102 reconstructed brightness temperatures by scattered celestial 103 radiation over the ocean and 2) to propose a method for the 104 proper detection of and correct for this sky glitter within the 105 SMOS ocean surface salinity retrieval algorithm.

106 For these two purposes, a sky brightness temperature map 107 at L-band was generated for SMOS based on an existing all108 sky continuum map using an approach similar to [2], and the 109 method used to build this map is reviewed in Section II. Missing 110 data in the vicinity of Cassiopeia A and other strong sources in 111 the continuum map can potentially lead to underestimation of 112 the reflected sky noise, particularly over smooth sea surfaces, 113 and to address this issue, we derived an error map by using 114 higher resolution surveys to identify the locations and bright115 ness temperatures of sources that may introduce substantial 116 errors associated with estimating the downwelling celestial 117 radiation from the continuum map alone. Since these localized 118 strong sources might require the use of very high-resolution 119 grids when applying the modeling methodology developed in 120 Part I, we evaluated the impact of resolution on the numerical 121 scattering calculations and determined an acceptable discretiza122 tion of the celestial noise map.

123 In Section III, we formulate expressions for the rough surface 124 scattered celestial radiation incident at the SMOS antenna. The 125 interferometric nature of the Microwave Imaging Radiometer 126 by Aperture Synthesis (MIRAS) results in a formulation that is 127 distinct from that for a real aperture radiometer. In developing 128 these expressions, we consider, in turn, simplifications that are 129 obtained by assuming that the sea surface is perfectly smooth 130 and approximating the synthetic antenna weighting function by 131 an isotropic function in director cosine (DC) coordinates.

132 Given the potential for scattered celestial noise to introduce 133 seasonal and regional biases in retrieved surface salinity, we ex134 amined the seasonality and spatial distribution of the expected 135 celestial sky glitter contamination for SMOS by performing 136 a series of monthly orbit propagations in which we collected 137 dwell lines, i.e., sets of multiangular scene brightness temper138 atures at a fixed location on Earth, over a fixed Earth grid 139 that spans one complete orbit. The scattered celestial radiation 140 was calculated for one orbit per month over a one-year period 141 using idealized descriptions of the ocean surface state. Results 142 of these calculations are presented in Section IV. The SMOS 143 configuration, with its sun-synchronous orbit and large field 144 of view (FOV), provides reconstructed brightness temperatures 145 over a large range of incidence and azimuth angles (and there- fore a large range of specular sky locations) at each point on 146 Earth, so that a large portion of the sky will contribute to 147 the contamination at any given time. Moreover, given the sun- 148 synchronous nature of the SMOS orbit, the celestial sky glitter 149 contamination is a function of time with a distinct annual cycle. 150 This is distinct from the situation with the Aquarius/SAC-D 151 mission, for which the set of all specular sky locations (or 152 specular projection of the FOV) at any given time of year is 153 a large closed loop on the celestial sphere. For SMOS, the 154 error associated with assuming a perfectly smooth sea surface 155 may exceed $0.1 \mathrm{~K}$ over large portions of the measurements 156 in an orbit. Both the contamination and the potential error 157 associated with assuming a perfectly flat surface are greatest 158 for the descending passes from August to October.

Given the potential for significant impact of the rough ocean 160 surface and the large computational burden associated with the 161 scattering calculations, in Section V, we propose a practical 162 correction and flagging strategy that may be used in a salinity 163 retrieval algorithm. In Section VI, we summarize the results and 164 briefly discuss potential sources of error as well as validation 165 issues for the proposed celestial sky glitter correction.

\section{Generation of An L-Band Sky Map to Be USED FOR SMOS DATA PROCESSING}

Three components are required to build a map of the sky 169 emission at L-band [2].

1) The cosmic microwave background (approximately a 171 constant value of $2.725 \mathrm{~K}$ ).

2) The neutral hydrogen line (HI in astronomer's shorthand): 173 this strong emitting line is centered at $1420.4058 \mathrm{MHz} 174$ and is spread over a finite band by an additional Doppler 175 shift. In surveys of the continuum, this source is usually 176 rejected by a stopband filter.

3) The continuum at $\sim 1.4 \mathrm{GHz}$, which originates from a 178 variety of emission mechanisms (other lines than HI, syn- 179 chrotron, free-free, thermal, blended emission of discrete 180 radio sources, ...).

The final merged map to be used with SMOS, which is 182 termed here as the "nominal map," is expressed in the Besselian 183 Epoch B1950 [4], and in what follows, we display the results 184 (and perform the scattering calculations) in an equatorial coor- 185 dinate system (with coordinates given by right ascension and 186 declination) in this reference frame.

\section{A. Main Sources of Data}

To provide coverage of the whole sky, measurements ob- 189 tained by different instruments situated in both the Northern 190 and Southern Hemispheres must be combined. This requires 191 extensive data collection and calibration to ensure sufficient 192 data quality. The merging of these data sets requires cross cali- 193 bration and consideration for differences in instrument angular 194 resolutions. This work was conducted by experts in the field of 195 radio astronomy, and the maps introduced here are based upon 196 products produced by these experts.

1) Continuum: The data set identified here is a combination 198 of the Northern Sky survey made with the Stockert radio 199 


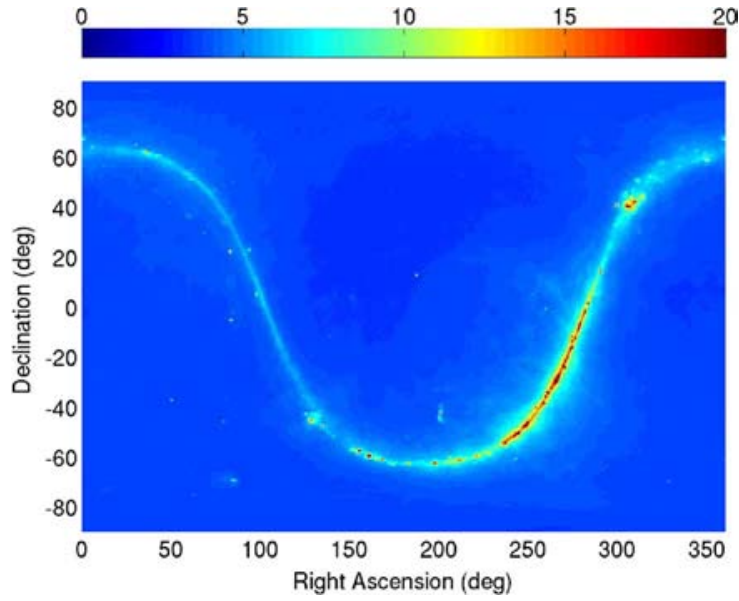

Fig. 1. Reich and Testori continuum map. Dark blue is for $0 \mathrm{~K}$, and red is for $20 \mathrm{~K}$.

200 telescope [5]-[7] and the Southern Sky survey made with the 201 radio telescope of the Instituto Argentino de Radioastronomia 202 (IAR) [8]. When the bandwidth of the receiver was overlapping 203 the HI emission, a stopband filter centered over the HI line 204 and $2 \mathrm{MHz}$ wide was applied to the measurement to reject it. 205 Data were sampled with a $0.25^{\circ}$ resolution in both declination 206 and right ascension (equatorial coordinates, B1950 system). 207 The sensitivity (defined as three times the root-mean-square 208 brightness temperature noise) of the merged data set is $0.05 \mathrm{~K}$. 209 In the following, this data set will be referred to as the Reich 210 and Testori map (Fig. 1).

211 It is assumed that the "continuum" radiation, with unpolar212 ized brightness temperature $T_{\text {cont }}$, is broadband and therefore 213 does not vary appreciably within the SMOS band. Thus, data 214 from surveys made at slightly different center frequencies and 215 with slightly different bandwidths may be directly combined. 216 The continuum data set includes the constant $2.725 \mathrm{~K}$ cosmic 217 background radiation.

218 2) Hydrogen Line: To account for the hydrogen line emis219 sion, we used the Leiden-Argentina-Bonn (LAB) survey [9]. 220 The LAB survey contains the final data release of obser221 vations of $21-\mathrm{cm}$ emission from galactic neutral hydrogen 222 over the entire sky and is a merged product based on the 223 Leiden-Dwingeloo survey of the sky north of $-30^{\circ}[10]$ and 224 the IAR survey of the sky south of $-25^{\circ}$ [11], [12]. The 225 source velocities away from the Earth range from -450 to $226+400 \mathrm{~km} \cdot \mathrm{s}^{-1}$ and are resolved in the data to $1.3 \mathrm{~km} \cdot \mathrm{s}^{-1}$. 227 The root-mean-square error of the brightness temperatures in 228 the merged data set is $0.07-0.09 \mathrm{~K}$ (for each $1.3 \mathrm{~km} \cdot \mathrm{s}^{-1}$ layer). 229 Data were sampled with a $0.5^{\circ}$ resolution in both latitude and 230 longitude (in galactic coordinates). Hereinafter, this data set 231 will be referred to as the HI map.

232 3) Integration of HI Into the Continuum Map: As men233 tioned earlier, the continuum signal is broadband, with almost 234 constant brightness temperature $T_{\text {cont }}$ throughout the SMOS 235 bandwidth. By contrast, the hydrogen line emission exists only 236 over a very narrow band, but MIRAS measures radiation over 237 a bandwidth $B_{\mathrm{SMOS}}$ of $19 \mathrm{MHz}$ that includes the HI line $238(1420.4058 \mathrm{MHz})$ so that this narrow source must be integrated 239 into the continuum map. The merged all-sky map provided by

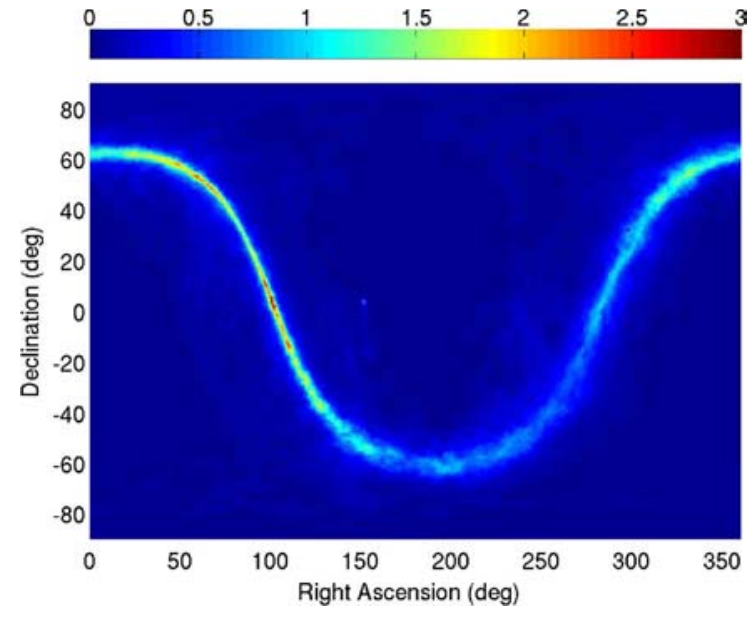

Fig. 2. HI map rescaled over SMOS bandwidth. Dark blue is for $0 \mathrm{~K}$, and red is for $3 \mathrm{~K}$.

Reich and Testori includes both $T_{\text {cont }}$ and the constant $2.725 \mathrm{~K} 240$ cosmic microwave background $T_{\mathrm{CMB}}$. The HI data [9] do not 241 include $T_{\mathrm{CMB}}$ (Fig. 2).

To derive the HI line contribution over the SMOS bandwidth 243 from HI line velocity range data, we used a Doppler relation 244 between velocity range and frequency shift. The HI line fre- 245 quency is $f_{0}=1420.4058 \mathrm{MHz}$, and the Doppler shift is given 246 by $f=f_{0}(c /(c+\nu))$, where $c$ is the speed of light and $\nu$ is the 247 relative speed of the source away from the Earth. The stopband 248 filter applied to the Reich and Reich measurements is centered 249 on $f_{0}$ and is $B_{\mathrm{HI}}=2 \mathrm{MHz}$ wide. This corresponds to outward 250 velocities ranging from -211.2 to $+211.4 \mathrm{~km} \cdot \mathrm{s}^{-1}$. Over this 251 bandwidth, the contribution of HI signal is

$$
\tilde{T}_{\mathrm{HI}}=\frac{1}{422.6 \mathrm{~km} \cdot \mathrm{s}^{-1}} \int_{-211.2 \mathrm{~km} \cdot \mathrm{s}^{-1}}^{211.4 \mathrm{~km} \cdot \mathrm{s}^{-1}} T_{\mathrm{HI}}(\nu) d \nu .
$$

Finally, the resulting sky noise to be considered for SMOS is

$$
T_{\mathrm{sky}}=T_{\mathrm{CMB}}+T_{\mathrm{cont}}+\tilde{T}_{\mathrm{HI}} \frac{B_{\mathrm{HI}}}{B_{\mathrm{SMOS}}} .
$$

\section{B. Gaps in the Continuum Survey: Use of Alternative Surveys 254 and Source Catalogs for Missing Data Integration

The Reich and Testori continuum survey is not complete and 256 contains regions with inadequate coverage. The most prominent 257 such region is Cassiopeia A, where the high flux prevented 258 accurate measurement using standard procedures. In addition, 259 highly localized strong sources are not properly taken into 260 account in the continuum survey. Higher resolution surveys that 261 can alleviate this problem by providing auxiliary $1.4-\mathrm{GHz}$ flux 262 measurements for these problematic areas are available. These 263 data sets usually come in two forms.

1) Higher resolution local sky maps where for a given 265 area of the sky a radio flux is associated to each [right 266 ascension, declination] cell. This enables an assessment 267 of the slow variations of the background flux when it 268 


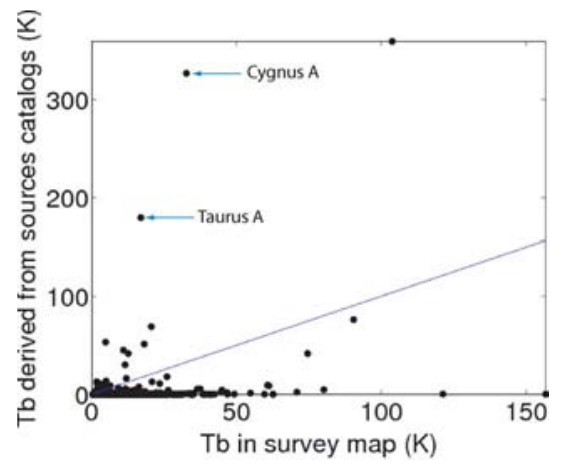

Fig. 3. Comparison between brightness temperatures derived from the individual source catalogs (NVSS + Parkes) and brightness temperatures extracted from the survey maps (i.e., a combination of the merged data from Reich and Testori, the rescaled HI line data from the survey in [9], and the Effelsberg survey data in the vicinity of Cassiopeia A). The diagonal line shows one-toone correspondence.

results from the combination of minor sources that cannot be individually identified. Once rescaled and converted to the proper geometry, these data sets can be used to patch the continuum map where data are missing.

2) Source catalogs that provide flux measurements for specific strong sources with small angular extents. These data sets can be useful to identify strong sources in otherwise quiet areas of the sky.

277 In the case of strong sources of small angular extent, it is diffi278 cult to determine whether they are properly taken into account 279 in the full sky survey map. To evaluate the extent to which 280 strong sources are properly accounted for in the continuum 281 map, a map of strong sources was generated from L-band 282 source catalogs [13], [14], and we computed the corresponding 283 brightness temperatures that would be collected by the Stockert/ 284 IAR radio telescopes (the ones that were used to generate the 285 Reich and Testori map). These source data were obtained from 286 both the [NRAO (National Radio Astronomy Observatory) 287 VLA (Very Large Array) Sky Survey (NVSS)] [14] (Northern 288 Hemisphere) and the Parkes [13] (Southern Hemisphere) cata289 logs. Only sources stronger than 0.3 Jy were considered, since 290 smaller fluxes would introduce less than $0.015-\mathrm{K}$ error in the 291 Reich and Testori map.

292 The resulting brightness temperatures were compared with 293 the combination of the continuum and HI maps. Fig. 3 shows 294 that most sources exhibit a brightness temperature that does 295 not exceed the corresponding continuum value (which is gen296 erally the case when the sources are embedded in regions of 297 strong emission that dominate the total signal within the rela298 tively large beam of the telescope). Nevertheless, some strong 299 sources, such as Cygnus A and Taurus A, can be identified. 300 Fig. 4 shows the locations of the sources for which the fluxes 301 are underevaluated in the nominal sky map. Most discrepancies 302 in the flux are quite small and are expected to be strongly 303 reduced when integrated over the SMOS synthetic beam. The 304 strongest discrepancies occur in the vicinity of Cygnus A and 305 Cassiopeia A. The nominal sky map generated for SMOS was 306 not corrected for these strong sources; instead, a separate sky 307 map that contains only the strong source brightness temper308 atures averaged to the Stockert/Testori beamwidth was devel-

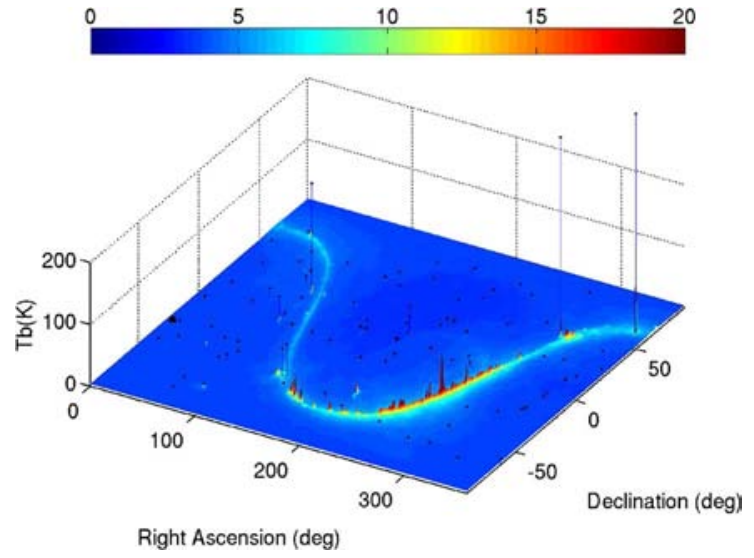

Fig. 4. Strong sources superimposed on the nominal SMOS sky map derived from the survey data of Reich and Testori, the rescaled HI line data from the survey in [9], and the Effelsberg survey data in the vicinity of Cassiopeia A Only sources that exhibit a brightness temperature (for a 35-arcmin beam) larger than that in the nominal sky map are displayed.

oped. As in [2], we do not account for possible polarization in 309 either the nominal or the strong source maps.

\section{Impact of Strong Sources}

To quantify the maximum expected impact of strong point 312 sources, we calculated the scattered signal along cross sections 313 through Cassiopeia A, where the strongest sources are located, 314 at both full $\left(0.25^{\circ} \times 0.25^{\circ}\right)$ and reduced-resolution $\left(3.75^{\circ} \times 315\right.$ $3.75^{\circ}$ ) celestial grid spacings for both the nominal and strong 316 source maps. The reduced-resolution map was obtained by 317 applying an energy flux-conserving averaging operator, which 318 is described in the Appendix, to the full-resolution map. The re- 319 sults are shown in Fig. 5. To provide an indication of the spatial 320 extent of the bistatic scattering cross sections on the celestial 321 map, in Fig. 5(a), we overlay on the total celestial noise map 322 (centered on Cassiopeia A) the weighting function associated 323 with the scattering cross sections for a representative scattering 324 calculation at a wind speed of $7 \mathrm{~m} \cdot \mathrm{s}^{-1}$. The total scattered 325 signal in the direction of the instrument is obtained by in- 326 tegrating the product of this weighting function and the sky 327 brightness temperatures over the sky map. The downwind di- 328 rection $\varphi_{w}$ relative to the scattering azimuth $\phi_{s}$ (both defined to 329 be positive counterclockwise from due east) is $\varphi_{w}-\phi_{s}=0^{\circ} .330$ This weighting function has been normalized to a maximum 331 of unity, and contours are shown at $0.1,0.3,0.5,0.7$, and 332 0.9 . Clearly, the weighting function extends well beyond the 333 localized strong source, so it is expected that the impact of such 334 a source should be small. In Fig. 5(b), we show the scattered 335 unpolarized signal $\left(1 / 2\left(T_{v}+T_{h}\right)\right)$ along a cross section at 336 constant declination in the celestial sphere, considering only 337 the strong source map. In this cross section, the wind speed 338 is fixed at $7 \mathrm{~m} \cdot \mathrm{s}^{-1}$, the specular declination is $58.25^{\circ}$, the 339 scattered field incidence angle is $0^{\circ}$, and the specular right 340 ascension ranges from $-40^{\circ}$ to $+20^{\circ}$. The resulting glitter is 341 shown for both the Kirchhoff approximation (KA) [15] and the 342 first-order small slope approximation (SSA-1) [16] scattering 343 models described in [3] and for both the full-resolution and 344 reduced-resolution maps. The resulting glitter never exceeds 345 

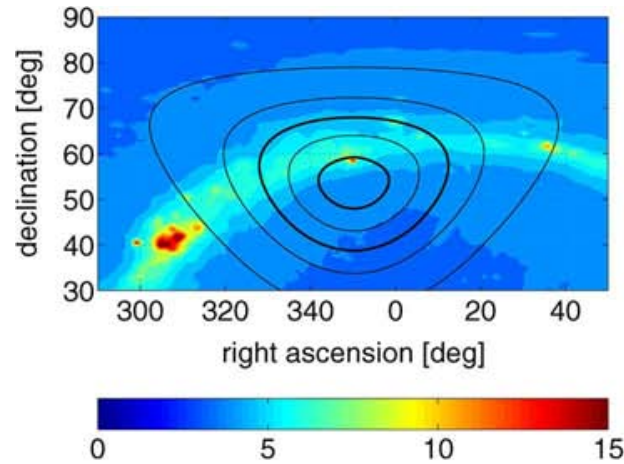

(a)

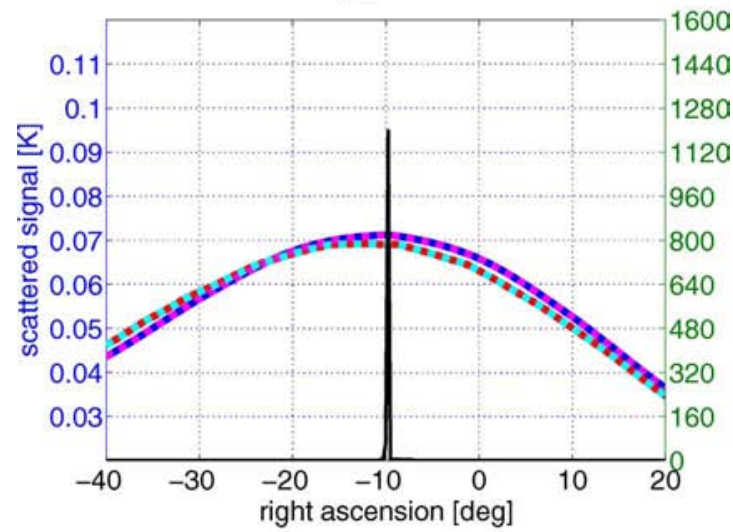

(b)

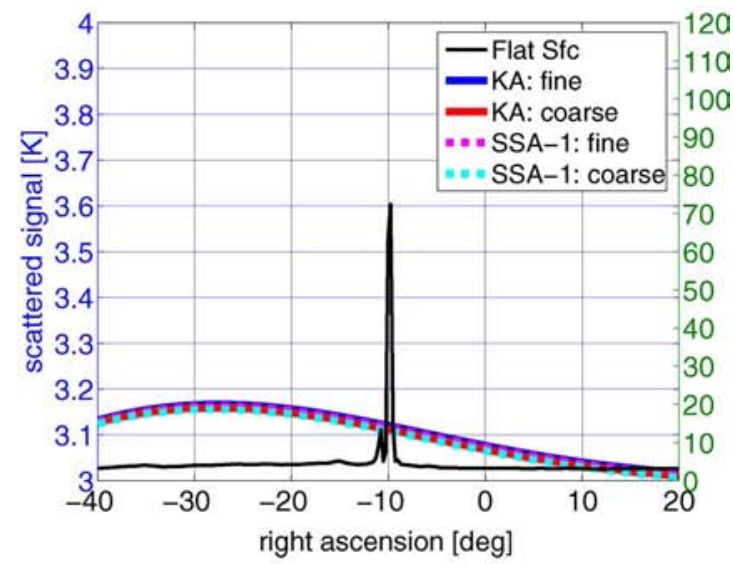

(c)

Fig. 5. (a) Incident unpolarized celestial noise. Overlaid is the bistatic scattering weighting function (discussed further in the text) for horizontal polarization for a specular point defined by $\left(\alpha_{s}=350.25^{\circ}, \delta_{s}=58.5^{\circ}\right)$ (Kirchhoff model; Kudryavtsev wave spectrum), which is normalized to unit amplitude. Thin contour lines correspond to the normalized levels $(0.1,0.3,0.7)$, and thick contour lines correspond to the normalized levels $(0.5,0.9)$. (b) Cross section, at constant declination, of the unpolarized $\left(1 / 2\left(T_{v}+T_{h}\right)\right)$ scattered radiation from strong sources as determined using the KA [15] and SSA-1 [16] electromagnetic models and the Kudryavtsev equilibrium ocean surface wave spectrum. Curves are defined in the inset of (c). The specularly reflected signal (solid black curve) is scaled to the right axis, whereas the scattered signals are scaled to the left axis. (c) Same as in (b) except for the nominal celestial noise map without strong sources. The scattered signal is evaluated at an incidence angle of $\theta_{s}=0^{\circ}$ and the incidence plane orientation angle $\psi_{u h}=0^{\circ}$. The surface wind speed is $u_{10}=7 \mathrm{~m} \cdot \mathrm{s}^{-1}$; the downwind direction relative to the scattering azimuth is $\varphi_{w}-\phi_{s}=0^{\circ}$. The maximum incident signal in the strong source map exceeds $1700 \mathrm{~K}$, while in the nominal map the maximum signal in the vicinity of this strong source is approximately $150 \mathrm{~K}$ [with a maximum at a slightly displaced position of $\left.\left(\alpha_{s}=350.25^{\circ}, \delta_{s}=58.25^{\circ}\right)\right]$. Units are in kelvin. about $0.07 \mathrm{~K}$, and the differences between solutions based on 346 the two scattering models and two map resolutions are negligi- 347 ble, even for this source with a peak brightness temperature ex- 348 ceeding $1100 \mathrm{~K}$.

Results from the same calculation with the nominal map are 350 shown in Fig. 5(c), where it is seen that differences between 351 resolutions and models are negligible, just as with the strong 352 source map. Notably, the maximum in the glitter does not 353 coincide with the strongest source, which can be understood 354 by examination of Fig. 5(a), which shows that the maximum 355 scattered signal can be expected when the overlap between the 356 weighting function and the galactic equator reaches a max- 357 imum. This maximum occurs for a specular point displaced 358 significantly from Cassiopeia A toward smaller right ascension. 359

\section{Celestial Sky Glitter Contribution AT THE SMOS INSTRUMENT LEVEL}

In Part I, we examined the rough surface scattered noise with- 362 out reference to a specific observational platform. Although the 363 results of that study provide an indication of expected depen- 364 dence of the scattered celestial sky glitter on both geophysical 365 variables (such as wind speed and direction) and observational 366 geometry, they do not address the impact of antenna patterns 367 and the potential systematic contamination associated with 368 orbit and viewing geometry. In this section, we consider the 369 impact of the SMOS synthetic antenna weighting function on 370 the resulting contamination.

\section{A. General Formulation}

Considering the simple case of unpolarized celestial radia- 373 tion with scalar brightness temperature $T_{\text {sky }}$ and assuming a 374 simple exponential model for attenuation on both downward 375 and upward paths, it was shown in Part I that the total antenna 376 temperature Stokes component $p$ (where $p$ corresponds to either 377 horizontal or vertical polarization) associated with rough sea 378 scattered celestial radiation is

$$
\begin{aligned}
\overline{\mathbf{T}}_{p}^{a}= & \frac{1}{\Omega_{a}} \int_{\Omega_{a}} \frac{\left(\mathbf{G M}_{\alpha}\right)}{4 \pi \cos \theta_{s}} e^{-a \sec \theta_{s}} \\
& \times \int_{\Omega_{0}\left(\Omega_{a}\right)}\left[\sigma_{p p}\left(\Omega_{0}\right)+\sigma_{p q}\left(\Omega_{0}\right)\right] e^{-a \sec \theta_{0}} T_{\mathrm{sky}}\left(\Omega_{0}\right) d \Omega_{0} d \Omega_{a}
\end{aligned}
$$

where the factor in front of the outermost integral normalizes 380 the antenna gain pattern $\mathbf{G}$. As detailed in Part $\mathbf{I}, \mathbf{M}_{\alpha}$ is a 381 composite transformation matrix accounting for polarization 382 basis and Faraday rotation. The scattered field incidence angle 383 is $\theta_{s}$, and $\sigma_{p q}$ are the normalized bistatic scattering cross 384 sections of the rough sea surface defined using the forward 385 scattering alignment polarization basis convention as discussed 386 in Part I and in [17]; $\Omega_{a}$ refers to the solid angle domain 387 of integration over the antenna pattern and is associated with 388 antenna incident and azimuth angles $\theta_{a}$ and $\phi_{a}$, respectively; 389 $\Omega_{0}$ refers to the entire upper hemisphere of over which sky 390 radiation is incident at the target; $a$ is the zenith atmospheric 391 
392 attenuation. Note that the normalized bistatic scattering cross 393 sections used in this paper and in Part I differ from the bistatic 394 scattering coefficients $\gamma_{p q}$ used in [1] in that the normalized 395 scattering cross sections relate scattered wave energy flux 396 across the undisturbed scattering surface to the incident flux 397 in the incident wave propagation direction, whereas the bistatic 398 scattering coefficients relate the scattered energy flux across the 399 surface to the incident energy flux across the surface, so that $400 \sigma_{p q}=\cos \theta_{0} \gamma_{p q}$. This distinction is briefly discussed in [18]. 401 In the case of an interferometric instrument such as MIRAS, 402 we are concerned with modeling the reconstructed brightness 403 temperature rather than the conventional antenna temperature 404 as obtained from a real aperture radiometer. The reconstructed 405 brightness temperature in direction $\left(\theta_{a}, \phi_{a}\right)$ is still given by an 406 equation of the form (3), but the outermost integral over $\Omega_{a}$ 407 is evaluated over a rather narrow synthetic antenna weighting 408 function centered at synthetic boresight direction $\left(\theta_{a}, \phi_{a}\right)$, and 409 the real aperture antenna gain matrix $\mathbf{G}$ is replaced by a 410 synthetic antenna weighting function that, in general, depends 411 upon both the instrument and the image reconstruction method. 412 In what follows, we will use the terms "reconstructed brightness 413 temperatures" and "brightness temperature measurements" in414 terchangeably.

415 If we introduce instrument DC coordinates $(\xi, \eta)$

$$
\begin{aligned}
& \xi=\sin \theta_{a} \cos \phi_{a} \\
& \eta=\sin \theta_{a} \sin \phi_{a}
\end{aligned}
$$

416 then the SMOS synthetic antenna weighting function, which is 417 also called equivalent array factor (AF), may be written [19] as

$$
\begin{aligned}
\operatorname{AF}_{\mathrm{eq}}\left(\xi, \xi^{\prime}, \eta, \eta^{\prime}\right)= & \frac{\sqrt{3}}{2} d^{2} \sum_{m} \sum_{n} W\left(u_{m n}, v_{m n}\right) \\
& \times \tilde{r}\left(-\frac{u_{m n} \cdot \xi+v_{m n} \cdot \eta}{f_{0}}\right) \\
& \times e^{j 2 \pi\left(u_{m n} \cdot\left(\xi-\xi^{\prime}\right)+v_{m n} \cdot\left(\eta-\eta^{\prime}\right)\right)}
\end{aligned}
$$

418 where $W$ is the apodization function; $\tilde{r}$ is the fringe-washing 419 factor (FWF), which accounts for the spatial decorrelation 420 between antennas; $u, v$ are the baseline coordinates in the 421 frequency domain; $d$ is the dimensionless antenna element 422 spacing $(0.875) ; f_{0}$ is the central frequency (1413 MHz); $\xi, \eta$ 423 are the central (i.e., synthetic boresight) DC coordinates; and $424 \xi^{\prime}, \eta^{\prime}$ are the running DC coordinates. Defining $\mathcal{D}=\left\{\xi^{\prime}, \eta^{\prime}\right.$ : $\left.425 \xi^{\prime 2}+\eta^{\prime 2}<1\right\}$ as the domain of integration within the synthetic 426 beam and noting that

$$
d \Omega_{a}=\sin \theta_{a} d \theta_{a} d \phi_{a}=\frac{d \xi d \eta}{\cos \theta_{a}}=\frac{d \xi d \eta}{\sqrt{1-\xi^{2}-\eta^{2}}}
$$

427 the expression for the contribution of the polarized celestial 428 sky glitter to the reconstructed brightness temperature in the 429 Ludwig-3 polarization basis is given by

$$
\begin{aligned}
\overline{\mathbf{T}}_{p}^{a}(\xi, \eta)= & \iint_{\mathcal{D}} \frac{\mathrm{AF}_{\mathrm{eq}}\left(\xi, \xi^{\prime}, \eta, \eta^{\prime}\right)}{\sqrt{1-\left(\xi^{\prime}-\xi\right)^{2}-\left(\eta^{\prime}-\eta\right)^{2}}} \\
& \times\left[\mathbf{M}_{\alpha}\left(\xi^{\prime}, \eta^{\prime}\right) \mathbf{A}_{u}\left(\xi^{\prime}, \eta^{\prime}\right)\right] \tilde{\mathbf{T}}_{p}^{a}\left(\xi, \xi^{\prime}, \eta, \eta^{\prime}\right) d \xi^{\prime} d \eta^{\prime}
\end{aligned}
$$

where $\tilde{\mathbf{T}}_{p}^{a}\left(\xi, \xi^{\prime}, \eta, \eta^{\prime}\right)$ is the Stokes vector of the surface scat- 430 tered celestial noise in the target polarization basis, and $\mathbf{A}_{u} 431$ is an upward atmospheric attenuation matrix defined in Part I. 432 The variations in atmospheric attenuation and geometrical rota- 433 tion are sufficiently small within the narrow (approximately $3^{\circ}$ ) 434 synthetic beam that these factors may be approximated by their 435 values at the synthetic beam center, i.e., $(\xi, \eta)$, so that

$$
\begin{aligned}
& \overline{\mathbf{T}}_{p}^{a}(\xi, \eta) \simeq \mathbf{M}_{\alpha}(\xi, \eta) \mathbf{A}_{u}(\xi, \eta) \\
& \quad \times \iint_{\mathcal{D}} \frac{\mathrm{AF}_{\mathrm{eq}}\left(\xi, \xi^{\prime}, \eta, \eta^{\prime}\right)}{\sqrt{1-\left(\xi^{\prime}-\xi\right)^{2}-\left(\eta^{\prime}-\eta\right)^{2}}} \tilde{\mathbf{T}}_{p}^{a}\left(\xi, \xi^{\prime}, \eta, \eta^{\prime}\right) d \xi^{\prime} d \eta^{\prime} .
\end{aligned}
$$

As shown in [20], if one neglects the FWF, the AF may be 437 approximated by a rather narrow centrosymmetric function 438 that is independent of the location of the synthetic boresight 439 $(\xi, \eta)$ within the FOV. The following explicit formula has been 440 developed to approximate the actual AF with no FWF effect: 441

$$
\begin{aligned}
\operatorname{AF}_{\mathrm{eq}}\left(\xi, \xi^{\prime}, \eta, \eta^{\prime}\right) & \simeq F_{\mathrm{cs}}\left(\rho\left(\xi, \xi^{\prime}, \eta, \eta^{\prime}\right)\right) \\
& =\max \left\{0,\left[\frac{\sin k_{f} \cdot \rho}{k_{f} \cdot \rho}\right]^{k_{k}} \cdot \frac{1}{1+k_{g} \cdot \rho^{k_{h}}}\right\}
\end{aligned}
$$

where $\rho=\sqrt{\left(\xi^{\prime}-\xi\right)^{2}+\left(\eta^{\prime}-\eta\right)^{2}}$ is the distance in DC co- 442 ordinates, $k_{f}=73.30, k_{g}=524.5, k_{h}=2.1030$, and $k_{k}=443$ 1.4936. Throughout the rest of this paper, we refer to the 444 approximate AF expression (10) as the antenna weighting 445 function, i.e., WEF. If we adopt this approximate formulation 446 for the AF and assume that the downwelling sky radiation 447 is unpolarized, then the total contamination of reconstructed 448 brightness temperatures by scattered celestial sky glitter (3) 449 becomes

$$
\begin{gathered}
\overline{\mathbf{T}}_{p}^{a}(\xi, \eta) \simeq \frac{\mathbf{M}_{\alpha}(\xi, \eta)}{4 \pi \cos \theta_{s}(\xi, \eta)} e^{-a \sec \theta_{s}(\xi, \eta)} \iint_{\mathcal{D}_{\rho}} d \phi d \rho \frac{\rho F_{\mathrm{cs}}(\rho)}{\sqrt{1-\rho^{2}}} \\
\times \int_{\Omega_{0}}\left[\sigma_{p p}\left(\Omega_{0}\right)+\sigma_{p q}\left(\Omega_{0}\right)\right] e^{-a \sec \theta_{0}} T_{\text {sky }}\left(\Omega_{0}\right) d \Omega_{0} .
\end{gathered}
$$

In this equation, $\mathcal{D}_{\rho}=\left\{\rho, \phi:\left(\xi^{\prime}(\rho, \phi)\right)^{2}+\eta^{\prime}((\rho, \phi))^{2}<1\right\}$ is 451 the polar coordinate domain corresponding to the Cartesian 452 domain $\mathcal{D}$.

\section{B. Perfectly Smooth (Flat) Sea Surface Case}

When the sea surface is perfectly flat, the scattered celestial 455 sky glitter incident at the instrument in the surface polarization 456 basis reduces to

$$
\tilde{\mathbf{T}}_{p}^{f}=\left|R_{p p}^{(0)}\left(S, T_{s}, \theta_{s}\right)\right|^{2} T_{\mathrm{sky}}\left(\theta_{s}, \phi_{s}-\pi\right) e^{-a \sec \theta_{s}}
$$

where $R_{p p}^{(0)}\left(S, T_{s}, \theta_{s}\right)$ are the Fresnel reflection coefficients of 458 the flat sea surface with salinity $S$, physical surface temperature 459 $T_{s}$, incidence angle $\theta_{s}$, and linear polarization $p$. In this case, 460 
461 the contribution to the reconstructed brightness temperature 462 from the sky glitter incident at the antenna from direction $(\xi, \eta)$, 463 which is expressed in the instrument polarization basis, is

$$
\begin{aligned}
\bar{T}_{p}^{\mathrm{fa}}(\xi, \eta) \simeq & \left(\mathbf{M}_{\alpha}(\xi, \eta) \mathbf{A}_{u}(\xi, \eta)\right) \iint_{\mathcal{D}} \frac{\mathrm{AF}_{\mathrm{eq}}\left(\xi, \xi^{\prime}, \eta, \eta^{\prime}\right)}{\sqrt{1-\left(\xi^{\prime}-\xi\right)^{2}-\left(\eta^{\prime}-\eta\right)^{2}}} \\
& \times\left|R_{p p}^{(0)}\left(S, T_{s}, \theta_{s}\left(\xi^{\prime}, \eta^{\prime}\right)\right)\right|^{2} \\
& \times T_{\mathrm{sky}}\left(\xi, \eta, \xi^{\prime}, \eta^{\prime}\right) e^{-a \sec \theta_{0}\left(\xi^{\prime}, \eta^{\prime}\right)} d \xi^{\prime} d \eta^{\prime} .
\end{aligned}
$$

464 The Fresnel power reflection coefficients vary weakly over the 465 significant portion of the synthetic beam, so that

$$
\left|R_{p p}^{(0)}\left(S, T_{s}, \theta_{s}\left(\xi^{\prime}, \eta^{\prime}\right)\right)\right|^{2} \simeq\left|R_{p p}^{(0)}\left(S, T_{s}, \theta_{s}(\xi, \eta)\right)\right|^{2} .
$$

466 With this approximation, the celestial sky glitter contribution at 467 the SMOS antenna for a perfectly flat sea surface becomes

$$
\begin{gathered}
\bar{T}_{p}^{f a}(\xi, \eta) \simeq \mathbf{M}_{\alpha}(\xi, \eta) e^{-a \sec \theta_{s}(\xi, \eta)}\left|R_{p p}^{(0)}\left(S, T_{s}, \theta_{s}(\xi, \eta)\right)\right|^{2} \\
\quad \times \iint_{\mathcal{D}_{\rho}} \frac{\rho F_{\mathrm{cs}}(\rho)}{\sqrt{1-\rho^{2}}} T_{\mathrm{sky}}(\xi, \eta, \rho, \phi) e^{-a \sec \theta_{0}(\phi, \rho)} d \phi d \rho .
\end{gathered}
$$

468 If we ignore the downward atmospheric attenuation, then the 469 approximate formulation (15) for a perfectly smooth ocean 470 surface is particularly attractive from a processing point of 471 view because it allows the incorporation of the antenna pattern 472 effect by a presmoothing of the sky brightness temperature map 473 [i.e., the integral factor in (15)] with the idealized synthetic 474 antenna weighting function. The synthetic beam-weighted re475 flected celestial sky glitter contamination may then be obtained 476 for arbitrary viewing geometry with a simple interpolation from 477 the smoothed map followed by a matrix multiplication.

\section{C. Antenna Pattern Smoothing Versus Roughness Spreading}

479 Following (11), to properly account for the celestial glitter in 480 the presence of surface roughness, we must compute the scat481 tered noise throughout the synthetic beam and then integrate the 482 product of the weighting function $F_{\mathrm{cs}}$ and this scattered signal. 483 Such a computation is not practical, and hence, it is useful to 484 determine if we can avoid this averaging operation. To assess 485 the impact of the synthetic beam averaging, we selected a time 486 and satellite configuration such that a small but strong bright487 ness source exists inside the SMOS FOV. We then established 488 a fine mesh over a small portion of the FOV surrounding this 489 source $($ a $65 \times 65$ regular grid covering a $0.2 \times 0.2$ domain 490 in the antenna DC coordinates) and calculated the scattered 491 horizontally polarized signal (in the surface polarization basis) 492 at each grid point. The flat surface reflected signal with no 493 synthetic beam smoothing, which is shown in Fig. 6(a), exhibits 494 a maximum brightness temperature of approximately $50 \mathrm{~K}$. 495 The corresponding signal as smoothed by the synthetic beam, 496 which is shown in Fig. 6(b), is significantly smoother, with a 497 maximum brightness temperature of approximately $16 \mathrm{~K}$.
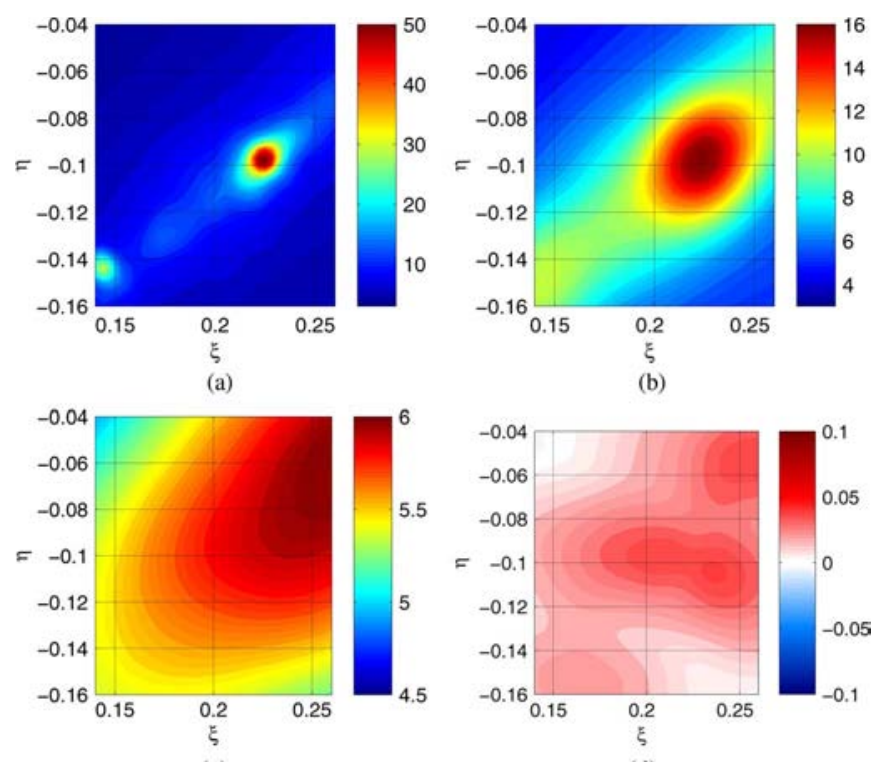

(b)

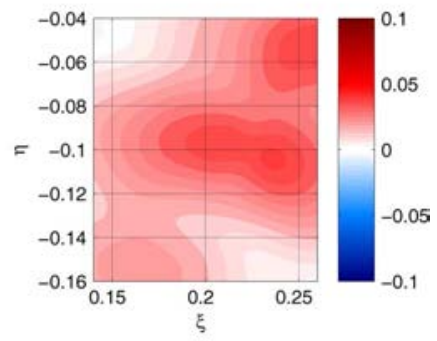

(d)

Fig. 6. (a) Flat sea surface specularly reflected signal at horizontal polarization. (b) Flat sea surface specularly reflected signal at horizontal polarization weighted by centrosymmetric WEF. (c) Bistatically scattered signal at horizontal polarization for a wind speed of $3 \mathrm{~m} / \mathrm{s}$. (d) Difference between scattered horizontally polarized celestial noise with and without weighting by the WEF. Units are in kelvin.

However, based on the scattering solutions obtained in this 498 paper, the impact of synthetic beam smoothing is far less than 499 that owing to the directional spreading of the radiation by the 500 roughened ocean surface, even at a wind speed of $3 \mathrm{~m} \cdot \mathrm{s}^{-1} 501$ [Fig. 6(c)]. This rough surface smoothing is sufficiently large 502 that applying the WEF smoothing to the scattering solutions 503 yields little change (generally less than about $0.05 \mathrm{~K}$ ), as shown 504 in Fig. 6(d). We conclude that the application of the WEF is 505 not necessary in the presence of surface roughness, so long as 506 this roughness is uniform within the synthetic antenna beam. 507 Although the WEF impact might be nonnegligible for surface 508 roughness at wind speeds lower than $3 \mathrm{~m} \cdot \mathrm{s}^{-1}$ or for highly 509 heterogeneous rough surfaces, in what follows, we neglect this 510 impact except for perfectly smooth surface conditions.

Without the WEF smoothing, the contribution of rough sur- 512 face scattered celestial sky glitter to the reconstructed bright- 513 ness temperatures reduces to

$$
\begin{aligned}
& \overline{\mathbf{T}}_{p}^{a}(\xi, \eta) \simeq \frac{\mathbf{M}_{\alpha}(\xi, \eta)}{4 \pi \cos \theta_{s}(\xi, \eta)} e^{-a \sec \theta_{s}(\xi, \eta)} \\
& \quad \times \int_{\Omega_{0}}\left[\sigma_{p p}\left(\Omega_{0}\right)+\sigma_{p q}\left(\Omega_{0}\right)\right] e^{-a \sec \theta_{0}} T_{\text {sky }}\left(\Omega_{0}\right) d \Omega_{0}
\end{aligned}
$$

and this equation is the basis for the results that follow.

\section{Annual Cycle of Celestial Sky Glitter CONTAMINATION FOR SMOS}

\section{A. Orbit Propagation and Dwell Line Generation}

Having established a reasonable approximation for the im- 519 pact of scattered celestial noise on the measurements, we 520 now quantify the impact of celestial sky glitter on SMOS 521 


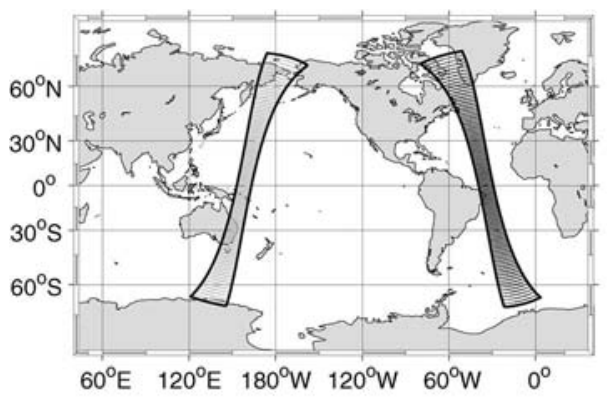

(a)

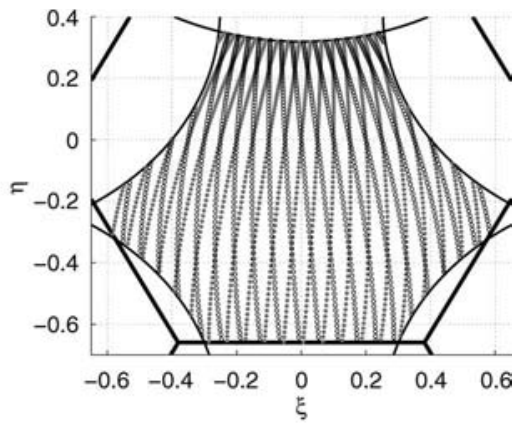

(b)

Fig. 7. (a) Fixed points on Earth at which we compute dwell lines for both ascending and descending swaths. (b) Projections of dwell lines in DC coordinates. Circles correspond to an ascending pass, while solid dots correspond to a descending pass.

522 measurements throughout the year. Given the sun-synchronous 523 nature of the SMOS orbit, the sky noise impact is expected to 524 exhibit a distinct annual cycle. To examine this annual cycle, 525 we performed a series of orbit propagations, with successive 526 orbits spaced roughly one month apart. Although we considered 527 both idealized and realistic geophysical conditions, here, we 528 present only the results from the idealized simulations (with 529 constant roughness conditions) to emphasize the impact of the 530 viewing geometry on the results. Introducing spatial and tem531 poral variabilities in the surface wind speed complicates the 532 interpretation of statistics and obscures the results, and the 533 expected behavior in variable wind conditions may be antici534 pated from the results presented in Part I. For the present sim535 ulations, bistatic scattering cross sections were evaluated at a 536 constant SSS of 35 psu and temperature of $15^{\circ} \mathrm{C}$. As discussed 537 in Part I, these two geophysical parameters will have a small 538 impact on diffuse scattering of celestial sky radiation. For com539 parison purposes, these surface conditions were also used to 540 estimate the contamination assuming a perfectly smooth ocean 541 surface. Moreover, to simplify the interpretation, we neglected 542 downward and upward atmospheric attenuation and only con543 sidered results for the first Stokes parameter (which is not 544 affected by the Faraday rotation on the upward path across the 545 ionosphere).

546 Orbit simulations were conducted using the same orbital 547 and instrument configuration anticipated for the actual satellite. 548 SMOS will be placed in a circular sun-synchronous low Earth 549 orbit at a mean flight altitude of $755 \mathrm{~km}$. The local time of 550 the ascending node will be 6:00 A.M., and the inclination of 551 the orbital plane will be $98.42^{\circ}$. In addition, the antenna array 552 plane will be tilted from the horizontal by $32^{\circ}$. To produce 553 one orbit simulation, we first established a fixed Earth grid by 554 propagating the satellite through one orbit at a time step of $24 \mathrm{~s}$ 555 and projecting onto the Earth's surface a set of points along a 556 cross-track line at $\eta=0.0$ in the instrument frame DC coordi557 nates. As illustrated in Fig. 7(a), this procedure establishes a $55821 \times 250$ point fixed grid $\mathbf{E}_{i j}$ on the Earth's surface, with 559250 rows (with index $i$ ) of 21 projected $\eta=0$ points (with 560 index $j$ ). Having established this grid, we then propagated the 561 satellite through the same orbit but with a 2.4-s time step, 562 producing a set of snapshots $\mathcal{S}_{k}$. At each of the $21 \times 250 \mathrm{grid}$ 563 points in a given SMOS FOV, we recorded parameters such 564 as the incidence and azimuth angles at target and the location in antenna frame $\left(\xi_{s}, \eta_{s}\right)$. The result is a grid of dwell lines, 565 where a dwell line at grid point $(i, j)$, which is denoted as $\mathcal{D}_{i j}, 566$ consists of a set of all $k$ for which $\mathcal{S}_{k}$ contains the point $\mathbf{E}_{i j} 567$ together with the corresponding set of positions in those snap- 568 shots, i.e.,

$$
\mathcal{D}_{i j}=\left\{k, \xi(i, j, k), \eta(i, j, k):(\xi(i, j, k), \eta(i, j, k)) \in \mathcal{S}_{k}\right\} .
$$

Fig. 7(b) shows examples of dwell lines in DC coordinates. 570

In the following experiments, we configured each orbit in the 571 monthly sequence of orbits so that the grid points do not change 572 location from one month to the next. Since we only consider 573 constant geophysical conditions, this has no significance be- 574 yond the fact that the geographical locations of the grid points 575 remain the same from one month to the next.

\section{B. Perfectly Smooth Sea Surface Contamination}

Before evaluating the impact of rough surface scattered sky 578 noise, we establish the impact of flat surface reflected noise as a 579 baseline. In Fig. 8, we show for each orbit the fraction of mea- 580 surements contaminated by unpolarized reflected celestial noise 581 $\left(1 / 2\left(T_{v}+T_{h}\right)\right)$ exceeding $4 \mathrm{~K}$. Most notable is the fact that the 582 reflected celestial sky noise is quite different for the ascending 583 and descending swaths, with generally larger contamination 584 in the descending swaths, which is to be expected since the 585 specular points for dwell lines on the descending swaths tend 586 to be aligned with the strongest portion of the noise originating 587 near the galactic equator. The contamination is greatest during 588 the northern hemisphere autumn when the specular points 589 tend to be nearest the galactic equator. A significant portion 590 of the dwell lines for the September 28 descending swath 591 have nearly $90 \%$ of their reconstructed brightness temperatures 592 contaminated by flat surface reflected noise exceeding 4 K. 593 The viewing geometry along any given dwell line is such that 594 the contamination patterns tend to be elongated in the FOV. 595 There is no time of the year that the entire swath is severely 596 contaminated. However, during certain times of the year, the 597 fraction of measurements suffering significant contamination 598 may be large. The contamination is also a function of latitude, 599 and the descending swaths in September tend to suffer from 600 more contamination in the Northern Hemisphere than in the 601 


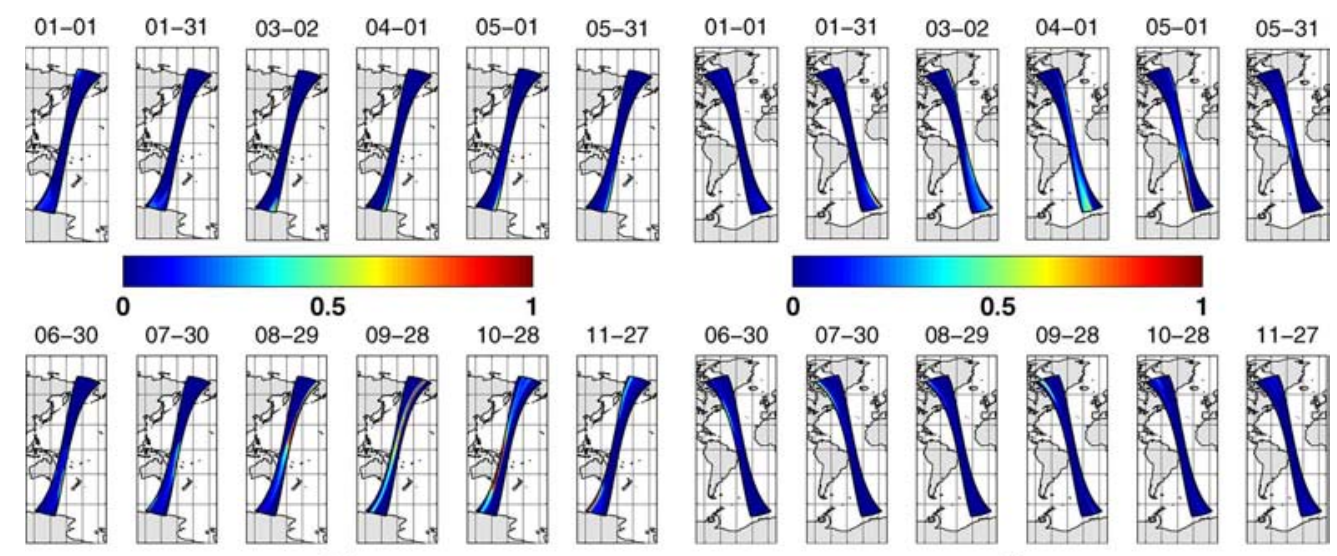

(a)

(b)

Fig. 8. Fraction of dwell line brightness temperature measurements contaminated by perfectly smooth sea surface reflected celestial noise $\left(1 / 2\left(T_{v}+T_{h}\right)\right)$ greater than $4 \mathrm{~K}$, for each month of the year. (a) Descending swaths. (b) Ascending swaths. The orbit dates (month-day) are indicated above each swath.

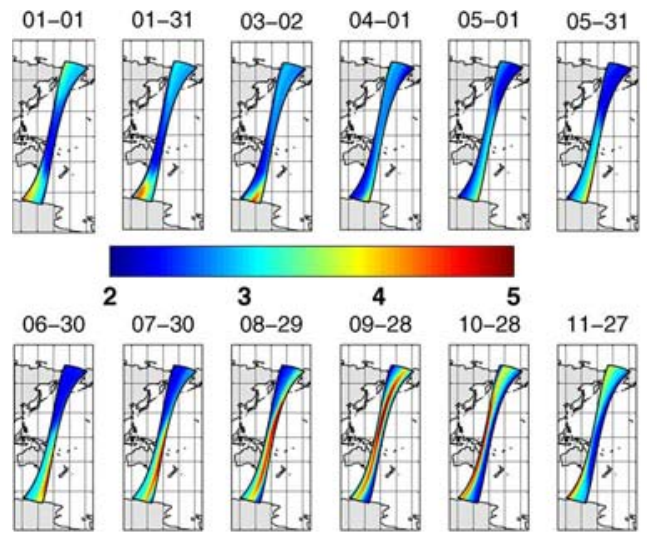

(a)

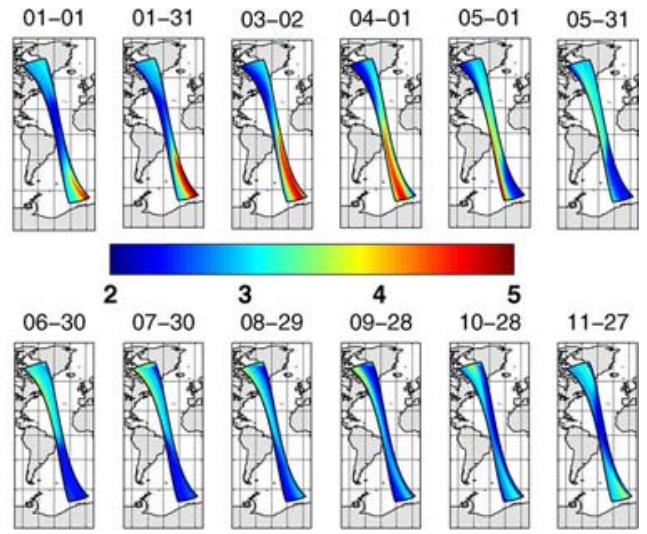

(b)

Fig. 9. Maximum unpolarized scattered celestial noise $\left(1 / 2\left(T_{v}+T_{h}\right)\right)$ over all measurements of each dwell line for (a) descending and (b) ascending swaths. The wind speed is $7 \mathrm{~m} \cdot \mathrm{s}^{-1}$, and the downwind direction is $0^{\circ}$. The Kudryavtsev wave spectrum and the KA scattering model are used to compute the scattered signal. Solutions are expressed in kelvin.

602 Southern Hemisphere. In October, the contamination maximum 603 shifts to the Southern Hemisphere.

\section{C. Rough Surface Contamination}

605 Although examination of the flat surface reflected celes606 tial noise provides some indication of expected contamination 607 patterns, it does not provide a realistic picture of the true 608 magnitude of the contamination, since at any given time only 609 about 5\% of the Earth's ocean surface is nearly perfectly 610 smooth [21]. As discussed in Part I, the differences between 611 the smooth surface reflected and rough surface scattered sig612 nals may be large, even at wind speeds below $7 \mathrm{~m} \cdot \mathrm{s}^{-1}$. In 613 this section, we examine the expected contamination pattern 614 for SMOS in idealized rough sea surface conditions with a 615 constant wind speed of $7 \mathrm{~m} \cdot \mathrm{s}^{-1}$ and downwind direction of $6160^{\circ}$. Using the Kudryavtsev equilibrium wave spectrum [22] and 617 the Kirchhoff scattering model (see Part I), we computed the 618 expected rough surface scattered celestial noise over the same 619 dwell lines considered in the previous section. In Fig. 9, we 620 show the maximum predicted unpolarized scattered sky noise $621\left(1 / 2\left(T_{v}+T_{h}\right)\right)$ for both descending and ascending passes 622 throughout the year. Both the spatial and temporal structures of the contamination are similar to those of the flat surface 623 contamination, but in the rough surface case, the patterns tend to 624 be smoother with significantly lower maximum contamination, 625 as one would expect from the results presented in Part I. 626 The strong maximum contamination first appears in late June 627 in the southern hemisphere and propagates across the FOV 628 toward higher $\xi$ in DC coordinates as time progresses. By 629 September 28, the peak contamination is situated near the 630 middle of the swath and nearly extends from pole to pole on 631 Earth. By late November, this maximum has shifted off the 632 right-hand side of the FOV.

In the ascending swaths, the time of year of maximum 634 contamination is different. The peak contamination, which is 635 slightly smaller in magnitude (approximately $4.7 \mathrm{~K}$ ) than for the 636 descending swaths, begins to enter the swath in early January 637 and propagates toward the west and north within the swath, 638 reaching the domain center in March, when it extends from the 639 South Pole to near the Earth's equator. By the end of May, the 640 peak has nearly left the swath.

The results for all of the orbits show that the time periods 642 of maximum contamination for the ascending and descending 643 swaths are nearly disjoint. At any given time of the year, either 644 the ascending or descending swaths, but not both, will suffer 645 


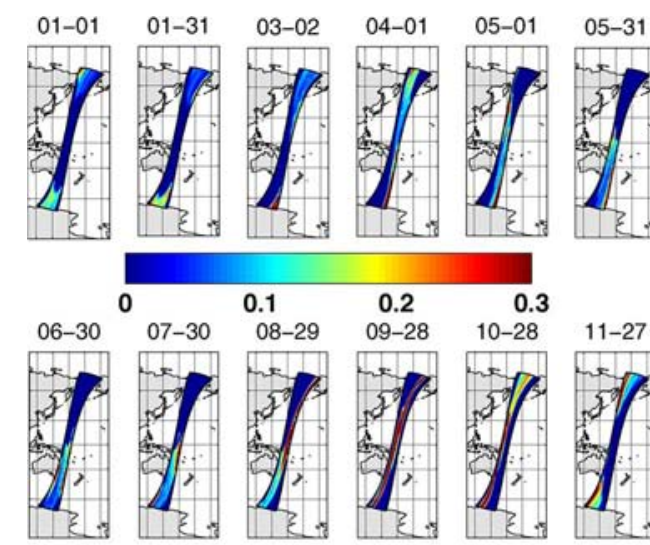

(a)

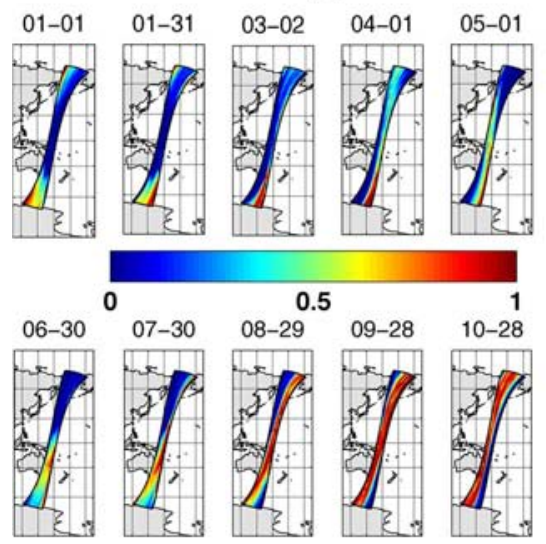

(c)

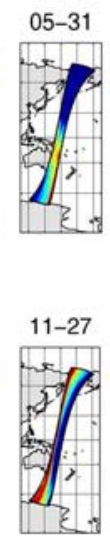

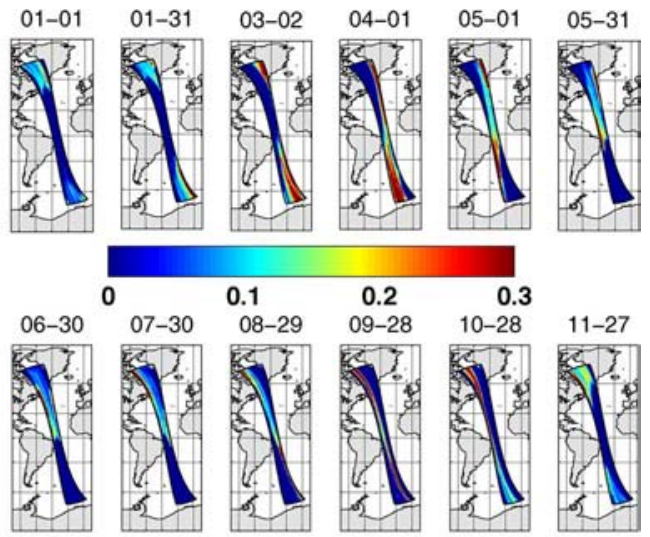

(b)
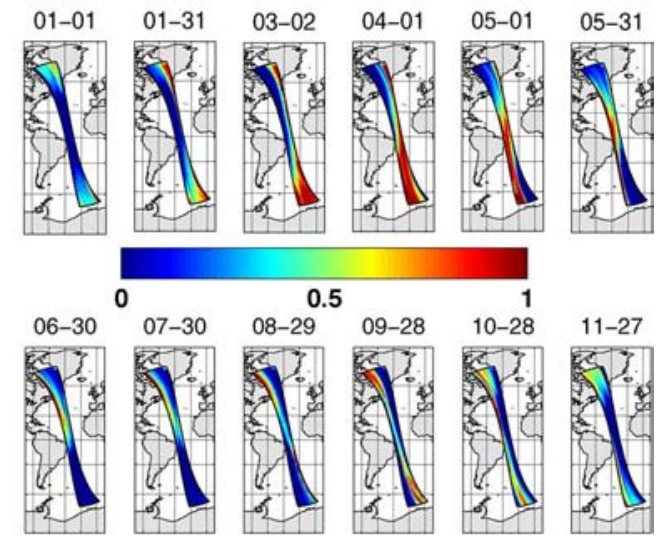

(d)

Fig. 10. (a) and (b) Fraction of dwell line brightness temperature measurements for which the difference between the unpolarized flat surface reflected signal $\left(1 / 2\left(T_{v}+T_{h}\right)\right)$ differs from the bistatically scattered signal by more than $0.5 \mathrm{~K}$, for descending and ascending swaths, respectively; (c) and (d): Same as in (a) and (b) except that the cutoff difference is $0.1 \mathrm{~K}$. The geophysical conditions are the same as in Fig. 9.

646 contamination. Given the relative ease with which contamina647 tion of reconstructed brightness temperatures by flat surface 648 reflected celestial noise may be evaluated, it is important to 649 determine if a more involved computation of the rough surface 650 scattered celestial noise will yield significantly different results. 651 Therefore, we assessed the overall difference between results 652 based on flat surface reflection and those based on rough surface 653 scattering calculations. Fig. 10(a) shows, at each dwell line of 654 the descending swaths, the fraction of measurements for which 655 the absolute difference between the perfectly smooth and rough 656 surface solutions exceeds $0.5 \mathrm{~K}$. The maxima in this fraction 657 generally exceed $10 \%$ and tend to coincide with the maxima in 658 the contamination. Similar results were obtained for the ascend659 ing swaths [Fig. 10(b)]. The fraction of measurements for which 660 the difference between the flat and rough solutions exceeds $6610.1 \mathrm{~K}$ approaches $100 \%$ for a substantial number of dwell lines, 662 as shown in Fig. 10(c) and (d). Given that the maxima in the 663 difference between the flat and rough surface solutions tend to 664 coincide with the maxima in the flat surface reflected noise, one 665 might hope to be able to develop a correction and error flagging 666 strategy based upon the flat surface solution. Unfortunately, the 667 differences between the flat and rough surface solutions do not 668 exactly coincide with the flat surface solution because the rough 669 surface scattering solutions depend strongly upon the spatial 670 structure of the source in the vicinity of the specular direction; therefore, it is not possible to determine a universal threshold 671 based on the flat surface solution alone.

\section{Processing Issues}

Given the significant and systematic impact of ocean surface 674 roughness on the contamination of reconstructed brightness 675 temperatures by celestial sky glitter, it is certainly desirable to 676 have a practical correction and flagging strategy for operation 677 purposes that incorporates the effect of surface roughness. Un- 678 fortunately, it is not practical to perform per-measurement inte- 679 grations of (16) to obtain scattering solutions, particularly given 680 the proposed SMOS level 2 iterative SSS inversion scheme in 681 which the surface wind speed is adjusted until convergence to a 682 solution for the salinity is achieved. The proposed solution for 683 SMOS level 2 processing involves precomputing the scattered 684 celestial noise for a range of wind speeds, incidence angles, 685 specular sky locations, and the incidence plane orientation 686 angle $\psi_{u h}$ introduced in Part I. The precomputed results are 687 stored in a lookup table from which solutions are obtained 688 during the salinity inversion procedure by interpolation. The 689 change of variables introduced in Part I involving the incidence 690 plane orientation angle $\psi_{u h}$ allows the separation of the impact 691 of viewing geometry at the target from the impact of specular 692 sky location, thereby enabling the creation of a lookup table 693 


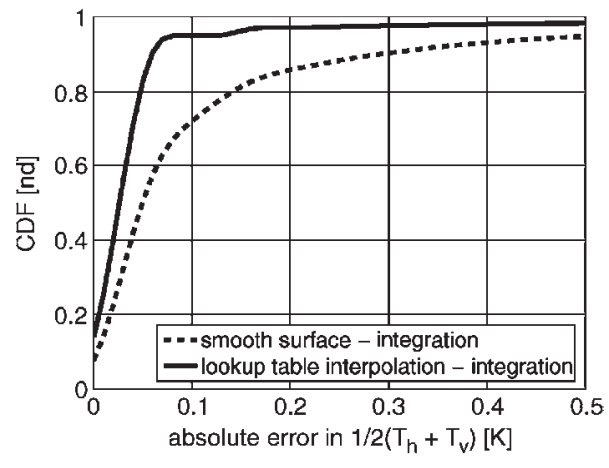

Fig. 11. Cumulative distribution function of the difference between $\left(1 / 2\left(T_{h}+T_{v}\right)\right)$ obtained by interpolation from the lookup table and by the per-measurement computation of the scattering solutions (solid curve). Cumulative distribution function of the difference between $\left(1 / 2\left(T_{h}+T_{v}\right)\right)$ obtained by use of the weighted flat surface reflection model and by the per-measurement computation of the scattering solutions (dashed curve). The differences are shown for the April ascending orbit only, and the geophysical conditions are the same as in Fig. 10. The Kudryavtsev wave spectrum and the KA electromagnetic scattering model are used for both the lookup table generation and the per-measurement integrations.

694 with practical discretizations in all dimensions. For the results 695 presented in this paper, we have generated scattered celestial 696 noise solutions for wind speeds of $3,5,7,10,15$, and $25 \mathrm{~m} \cdot \mathrm{s}^{-1}$ 697 on a regular $3.75^{\circ} \times 3.75^{\circ}$ grid in specular right ascension and 698 declination. The grid spacing in $\psi_{u h}$ is $22.5^{\circ}$, and the incidence 699 angles range from $0^{\circ}$ to $60^{\circ}$ by $5^{\circ}$ and from $60^{\circ}$ to $80^{\circ}$ by $10^{\circ}$. 700 To evaluate the performance of this lookup table solution, we 701 considered the same uniform geophysical conditions as in the 702 previous section and computed, for each measurement of each 703 dwell line, the difference between the solution obtained from 704 numerical integration of (16) and that obtained from multilinear 705 interpolation from the lookup table. The solid curve in Fig. 11 706 shows the cumulative distribution function of the absolute dif707 ference between these two computation methods for ascending 708 swath on April 1, when we expect the worst contamination for 709 ascending passes. The absolute difference in the unpolarized 710 signal $\left(1 / 2\left(T_{v}+T_{h}\right)\right)$ is less than $0.1 \mathrm{~K}$ in about $95 \%$ of 711 measurements, which is far better than that obtained with the 712 perfectly smooth surface solution. For comparison, the dashed 713 curve in the same figure shows the cumulative distribution func714 tion of the difference between the WEF-weighted flat surface 715 reflection model and the per-measurement integration results. 716 Similar results were found for the September 28 descending 717 swath. Therefore, the lookup table approach represents the 718 per-measurement integration with sufficient fidelity that it is 719 appropriate for use in an operation processor. Moreover, the 720 approach easily accommodates alternative scattering models. 721 The strong directional spreading effect of the rough ocean 722 surface permits the creation of the lookup table by integration 723 over a reduced resolution sky map with a grid spacing of $7243.75^{\circ} \times 3.75^{\circ}$ in right ascension and declination. Presently, the 725 rough surface scattering solutions are implemented for wind 726 speeds greater than $3 \mathrm{~m} \cdot \mathrm{s}^{-1}$. At zero wind speed, the WEF727 weighted smooth surface solution given in (15) is implemented 728 using the nominal high-resolution sky map (with $0.25^{\circ} \times 0.25^{\circ}$ 729 grid spacing and strong sources excluded). For nonzero wind 730 speeds below $3 \mathrm{~m} \cdot \mathrm{s}^{-1}$, surface roughness spectral descriptions are known to be inaccurate, and the approach we take to 731 estimate the celestial sky glitter contamination in this low wind 732 speed range is to linearly interpolate between solutions for the 733 perfectly smooth and $3 \mathrm{~m} \cdot \mathrm{s}^{-1}$ rough surfaces. Although this 734 approach lacks physical basis, it is proposed here as a practical 735 solution in the absence of an adequate rough surface statistical 736 description for low surface wind speeds. It is anticipated that the 737 algorithm will be refined based on SMOS data obtained after 738 launch.

\section{SUMMARY AND DISCUSSION}

In this paper, we have examined how the rough sea surface 741 scattering of L-band celestial sky radiation might affect SMOS 742 measurements.

We began by presenting the nominal celestial sky brightness 744 temperature map at L-band that was generated for SMOS 745 using an approach similar to that described in [2]. The nom- 746 inal map includes the appropriately integrated impact of the 747 hydrogen line emission, but the impact of highly localized 748 strong sources is neglected. Since omission of these strong 749 sources from this nominal sky map may introduce errors into 750 the scattering calculations, we also derived a map of strong 751 sources and their brightness temperatures using high-resolution 752 surveys. We found that, for wind speeds greater $3 \mathrm{~m} \cdot \mathrm{s}^{-1}$ and 753 for the two rough surface scattering models (KA and SSA-1) 754 considered in this paper, the scattered signals associated with 755 these localized strong sources are extremely small owing to 756 the directional spreading of the scattered signal by the rough 757 surface. Therefore, in the scattering calculations, we neglected 758 the impact of such sources.

Next, we established expressions for the expected signals at 760 the SMOS antenna array for both flat (perfectly smooth) and 761 rough seas. Using an approximate isotropic (in DC coordinates) 762 synthetic antenna weighting function (i.e., WEF), we obtained 763 expressions for the contribution of the scattered celestial sky 764 radiation to the total reconstructed brightness temperatures. In 765 theory, to properly assess the impact of celestial glitter in the 766 presence of surface roughness on the reconstructed brightness 767 temperatures, the scattered noise must be computed over the 768 instrument FOV and then integrated over the synthetic antenna 769 weighting function. Given the extreme computational burden of 770 this approach, we evaluated the impact of computing only the 771 synthetic boresight solution and avoiding the WEF integration 772 entirely. We found that, in general, the rough surface scattered 773 signal is sufficiently smooth that, even in the vicinity of a strong 774 (i.e., $50 \mathrm{~K}$ ) localized source, the scattered signal is not modified 775 by more than approximately $0.05 \mathrm{~K}$ by integration over the 776 WEF, so that this step may be avoided in rough ocean surface 777 conditions. Although the WEF impact might be nonnegligible 778 for surface roughness at wind speeds lower than $3 \mathrm{~m} \cdot \mathrm{s}^{-1}$ or 779 highly heterogeneous rough surfaces, we do not consider it, 780 except for the perfectly smooth surface conditions.

The sampling characteristics of the instrument are important 782 factors in determining the overall impact of scattered celestial 783 noise for a particular mission. Both the Aquarius/SAC-D and 784 SMOS satellites will maintain sun-synchronous orbits, so that 785 the specular reflection of the antenna pattern on the celestial 786 
787 sphere will slowly evolve with time, making one complete 788 cycle in a year. As compared with Aquarius/SAC-D, the large 789 FOV of MIRAS is associated with a much larger specular 790 domain in the celestial sphere, thus the reconstructed brightness 791 temperatures derived from MIRAS will suffer from a large 792 range of contamination at any given dwell line on Earth. The 793 results presented here indicate that the contamination exhibits 794 a strong seasonal cycle that is different for the ascending and 795 descending swaths. The largest contamination occurs in the 796 descending swath in September and October, when the specular 797 projection of the FOV is aligned with a strip of strong noise 798 in the vicinity of the galactic equator. The geometry of the 799 problem is such that contamination patterns tend to be elon800 gated in the along-track direction. Moreover, during one period 801 of time each year, the specular projections of dwell lines for 802 both the ascending and descending swaths will be aligned with 803 the galactic equator, and during this time period, contamination 804 will be most severe. Considering the flat sea surface reflected 805 signal alone, a significant fraction of the measurements in many 806 of the dwell lines will be contaminated by reflected celestial 807 noise exceeding $4 \mathrm{~K}$ in the descending swath during September 808 and October (and to a large extent in August and November). 809 Considering moderate wind speed conditions, a larger portion 810 of dwell lines will suffer from contamination in which surface 811 roughness modifies the flat surface specularly reflected signal 812 by more than $0.1 \mathrm{~K}$.

813 Given this potential for strong contamination in a large 814 fraction of measurements and the computational burden of the 815 rough surface scattering calculations, we examined a strategy 816 for computing the rough surface scattered signal using a pre817 computed lookup table expressed in terms of the specular sky 818 location, incidence angle, wind speed, and the incidence plane 819 orientation angle $\psi_{u h}$ introduced in Part I. For the monthly 820 orbits considered here, results obtained by interpolation from 821 the lookup table differ from per-measurement scattering calcu822 lations by less than $0.1 \mathrm{~K}$ in $95 \%$ of measurements for April and 823 September, during which we expect the worst contamination 824 and the largest impact of surface roughness.

825 In the monthly orbit calculations with a moderate surface 826 wind speed, only $70 \%$ of the rough surface scattered sig827 nals differ from the smooth surface counterparts by less than $8280.1 \mathrm{~K}$. Importantly, the numerically integrated scattered signals 829 are based on asymptotic scattering models, and the statistical 830 description of the rough surface is based on an idealized wave 831 model. Although we found that in the vicinity of the strongest 832 noise source the results obtained from two electromagnetic 833 models do not differ by more than $0.02 \mathrm{~K}$, the amplitudes and 834 phases of the wind direction dependence can exhibit large dif835 ferences between models. In addition, we have not considered 836 here the dependence of the results on the wave model, which 837 may have a significant impact on the contamination and its 838 relative wind direction dependence.

839 Finally, we have not considered polarized source radiation. 840 Recently, new surveys of linearly polarized radiation (i.e., the 841 third and fourth Stokes parameters) over the northern sky at a 842 frequency of $1.4 \mathrm{GHz}$ have become available [23]-[26]. These 843 maps reveal a highly variable polarized intensity that can reach $844500 \mathrm{mK}$ in magnitude (e.g., in the vicinity of the North Polar
Spur). This polarization, which is neglected in our formulation, 845 might impact the celestial noise contamination to an extent that 846 is significant for SSS retrieval. Unfortunately, incorporation of 847 this polarization complicates the formulation. In particular, the 848 polarimetric algorithm must account for polarization rotation 849 from the celestial basis to the usual target basis as well as 850 downward Faraday rotation. Generalizing the expression for the 851 total scattered brightness temperature in the antenna frame (3) 852 to the fully polarized case, we obtain

$$
\begin{aligned}
\overline{\mathbf{T}}_{p}^{a} & =\frac{1}{\Omega_{a}} \int_{\Omega_{a}} \frac{\left(\mathbf{G} \mathbf{M}_{\alpha}\right)}{4 \pi \cos \theta_{s}} e^{-a \sec \theta_{s}} \\
& \times \int_{\Omega_{0}\left(\Omega_{a}\right)} \mathbf{M}_{s}\left(\Omega_{a}, \Omega_{0}\right) \mathbf{M}_{\mathrm{fd}}\left(\Omega_{0}, t\right) \mathbf{M}_{\Psi} \mathbf{T}_{q} e^{-a \sec \theta_{0}} d \Omega_{0} d \Omega_{a}
\end{aligned}
$$

where $\mathbf{T}_{q}=\left(\mathbf{T}_{h}, \mathbf{T}_{v}, \mathbf{U}, \mathbf{V}\right)^{T}$ is the full Stokes vector of 854 incidence radiation, and $\overline{\mathbf{T}}_{p}^{a}$ is the Stokes vector of the 855 WEF-weighted signal in the instrument polarization basis. In 856 contrast with the formulation given in [3], which is appro- 857 priate for unpolarized sky radiation, $\mathbf{M}_{s}\left(\Omega_{a}, \Omega_{0}\right)$ is the fully 858 polarimetric Mueller matrix (with the obvious dependence on 859 the rough surface omitted). The incoming Stokes vector $\mathbf{T}_{q}$ is 860 transformed before scattering by a change in polarization basis 861 $\mathbf{M}_{\Psi}\left(\Omega_{0}\right)$ and the time-dependent Faraday rotation $\mathbf{M}_{\mathrm{fd}}\left(\Omega_{0}, t\right), 862$ which in turn depends upon the incident radiation direction. 863 The transformation matrix $\mathbf{M}_{\Psi}\left(\Omega_{0}\right)$ implicitly depends on the 864 target location and radiometer incidence and azimuth angles, 865 but these additional dependencies may be accounted for by the 866 incidence plane orientation angle $\psi_{u h}$ introduced in Part I, so 867 that more explicitly $\mathbf{M}_{\Psi}=\mathbf{M}_{\Psi}\left(\psi_{u h}, \Omega_{0}\right)$. Therefore, without 868 Faraday rotation, no additional difficulties are encountered in 869 the formulation of the lookup table. When we perform the 870 integration of the fully polarimetric scattering cross sections 871 over the upper hemisphere for each $\left\{\alpha_{s}, \delta_{s}, \theta_{s}, \psi_{u h}\right\}$, the set 872 of polarization basis rotations (one for each point in the upper 873 hemisphere integration) is completely determined. In practice, 874 source polarization may increase the sensitivity of the scattered 875 Stokes vector components to the orientation angle $\psi_{u h}$ and 876 therefore require a lookup table with finer resolution than for 877 the unpolarized case.

A more difficult problem involves the downward Faraday 879 rotation. Were it not for the time dependence in the Faraday 880 rotation, no additional problem would arise, since the ad- 881 ditional rotation could be incorporated into the polariza- 882 tion basis transformation as an additional rotation. However, 883 Faraday rotation is strongly time dependent, and this neces- 884 sitates further approximation to be able to use a pregener- 885 ated time-independent lookup table of scattered noise. One 886 possible approach is to approximate the downward Faraday 887 rotation by the time-dependent value evaluated only in the 888 specular direction. Unfortunately, in general, the scattering 889 matrix does not commute with the downward Faraday ro- 890 tation matrix, so that $\mathbf{M}_{s}\left(\Omega_{a}, \Omega_{0}\right) \mathbf{M}_{\mathrm{fd}}\left(\Omega_{0}, t\right) \neq \mathbf{M}_{\mathrm{fd}}\left(\Omega_{0}, t\right) 891$ $\mathbf{M}_{s}\left(\Omega_{a}, \Omega_{0}\right)$, and thus, it is not possible to bring $\mathbf{M}_{\mathrm{fd}}\left(\Omega_{0}, t\right) 892$ 
893 outside the integral, even with the time-dependent specular 894 approximation.

895 The effect of Faraday rotation is analogous to the effect 896 of the polarization basis rotation. Without Faraday rotation, 897 the polarization basis rotation is strictly a function of the 898 specular location and incidence plane orientation angle $\psi_{u h}$, so 899 that it will be accounted for implicitly. Unfortunately, Faraday 900 rotation is an additional (seventh) independent variable. If, 901 however, we neglect the portion of the $\psi_{u h}$ dependence related 902 to variations in the mapping from the upper hemisphere to the 903 sky, then we can adjust $\psi_{u h}$ by the Faraday rotation angle 904 to obtain a new pseudo-orientation angle $\tilde{\psi}_{u h}$ that effectively 905 accounts for Faraday rotation at the expense of properly ac906 counting for the (possibly more subtle) effect of sky noise 907 orientation on the upper hemisphere. According to the models 908 considered in this paper, the maximum peak-to-peak variability 909 (with respect to $\psi_{u h}$ ) in the scattered unpolarized signal was 910 on order of $0.5 \mathrm{~K}$; therefore, this approach may be worth 911 consideration.

912

913

914

915 Here, we describe the method that we used to generate a 916 reduced-resolution celestial map, which conserves the energy 917 flux of the full-resolution map.

918 Letting $\delta$ denote declination and $\alpha$ right ascension, the 919 original discrete celestial map provides data on a grid of cells 920 such that the brightness temperature field is piecewise constant 921 within each cell and has the form

$$
T_{\text {sky }}^{f}=T_{\text {sky }}^{f}\left(\delta_{0}^{f}+\left(j^{f}-1\right)(\Delta \delta)^{f}, \alpha_{0}^{f}+\left(k^{f}-1\right)(\Delta \alpha)^{f}\right)
$$

922 where $j^{f}$ and $k^{f}$ are positive integer indices that satisfy

$$
\begin{aligned}
& 1 \leq j^{f} \leq n_{\delta}^{f} \\
& 1 \leq k^{f} \leq n_{\alpha}^{f} .
\end{aligned}
$$

923 Here, the grid spacing is $(\Delta \delta)^{f}=0.25^{\circ}$ in declination and $924(\Delta \alpha)^{f}=0.25^{\circ}$ in right ascension. A reduced-resolution celes925 tial map was produced by applying an energy-flux-conserving 926 averaging operator $R(\cdot)$ to the original celestial map to 927 produce a celestial noise map on a low-resolution grid $928 \mathbf{G}_{r}\left(j^{c}(\Delta \delta)^{c}, k^{c}(\Delta \alpha)^{c}\right)$, where $(\Delta \delta)^{c}=\left(2 n^{c}+1\right)(\Delta \delta)^{f}$, and $929(\Delta \alpha)^{c}=\left(2 n^{c}+1\right)(\Delta \alpha)^{f}$. The integer rescaling factor $n^{c}$ is 930 set to 7 for this paper, which yields a 15 -fold increase in grid 931 spacing in right ascension and declination. The integer indices 932 of the coarse grid, i.e., $j^{c}$ and $k^{c}$, satisfy

$$
\begin{aligned}
& 1 \leq j^{c} \leq \frac{\left(n_{\delta}^{f}-1\right)}{\left(2 n^{c}+1\right)}+1 \\
& 1 \leq k^{c} \leq \frac{\left(n_{\alpha}^{f}-1\right)}{\left(2 n^{c}+1\right)}+1
\end{aligned}
$$

and the discrete low-resolution brightness temperature 933 field is

$$
\begin{aligned}
& T_{\text {sky }}^{c}\left(j^{c}, k^{c}\right)=\frac{1}{\mathcal{N}} \sum_{j^{f}=j_{0}^{f}}^{j_{1}^{f}} \sum_{k^{f}=k_{0}^{f}}^{k_{1}^{f}} \sin \left(\delta_{0}^{f}+j^{f}(\Delta \delta)^{f}\right) \\
& \times T_{\text {sky }}^{f}\left(\delta_{0}^{f}+\left(j^{f}-1\right)(\Delta \delta)^{f}, \alpha_{0}^{f}+\left(k^{f}-1\right)(\Delta \alpha)^{f}\right)
\end{aligned}
$$

where $T_{\text {sky }}^{f}$ is the (piecewise constant) fine grid brightness 935 temperature field, and $T_{\text {sky }}^{c}$ is the coarse grid field. The index 936 limits of summation over the fine grid may be expressed in 937 terms of coarse grid indices and the resolution reduction factor 938 $n^{c}$ by

$$
\begin{aligned}
& j_{0}^{f}\left(j^{c}\right)=1+\left(2 n^{c}+1\right)\left(j^{c}-1\right)-n^{c} \\
& j_{1}^{f}\left(j^{c}\right)=1+\left(2 n^{c}+1\right)\left(j^{c}-1\right)+n^{c} \\
& k_{0}^{f}\left(k^{c}\right)=1+\left(2 n^{c}+1\right)\left(k^{c}-1\right)-n^{c} \\
& k_{1}^{f}\left(k^{c}\right)=1+\left(2 n^{c}+1\right)\left(k^{c}-1\right)+n^{c} .
\end{aligned}
$$

The factor

$$
\mathcal{N}=\sum_{j^{f}=j_{0}^{f}}^{j_{1}^{f}} \sum_{k^{f}=k_{0}^{f}}^{k_{1}^{f}} \sin \left(\delta_{0}^{f}+j^{f}(\Delta \delta)^{f}\right)
$$

is a normalization factor for the averaging operator. The first 941 cell in the reduced grid (in both declination and right ascension) 942 is always aligned with the first cell in each dimension in the 943 original grid, and the celestial brightness temperature values 944 assigned to each of the reduced-resolution grid cells is the 945 weighted average of the brightness temperatures in all original 946 grid cells contained within the encompassing coarse grid cell. 947 In the averaging procedure, the weight given to a particular 948 fine grid cell is proportional to the solid angle subtended by 949 that cell.

\section{ACKNOWLEDGMENT}

The authors would like to thank S. Zine and D. Le Vine 952 for contributing to the SMOS sky map generation and the 953 anonymous reviewers for helping us improve the manuscript. 954 The centrosymmetric weighting function used in the SMOS 955 portion of the analysis was developed by P. Waldteufel.

\section{REFERENCES}

[1] S. H. Yueh, R. West, W. J. Wilson, F. K. Li, E. G. Njoku, and 958 Y. Rahmat-Samii, "Error sources and feasibility for microwave remote 959 sensing of ocean salinity," IEEE Trans. Geosci. Remote Sens., vol. 39, 960 no. 5, pp. 1049-1060, May 2001.

961

[2] D. M. Le Vine and S. Abraham, "Galactic noise and passive microwave 962 remote sensing from space at L-band," IEEE Trans. Geosci. Remote Sens., 963 vol. 42 , no. 1, pp. 119-129, Jan. 2004. 
[3] J. Tenerelli, N. Reul, A. A. Mouche, and B. Chapron, "Earth viewing L-band radiometer sensing of sea surface scattered celestial sky radiation. Part I: General characteristics," IEEE Trans. Geosci. Remote Sens., vol. 46, no. 3, pp. xxxx-xxxx, Mar. 2008.

[4] M. Sánchez-Nogales, F. Pirondini, and J. A. G. Abeytua, "Earth Explorer Mission CFI software: Mission conventions document," DEIMOS Space S.L., Madrid, Spain, Jul. 2003. Tech. Note.

[5] W. Reich, "A radio continuum survey of the northern sky at 1420 MHz-Part I," Astron. Astrophys., Suppl. Ser., vol. 48, pp. 219-297, Jul. 1982.

6] P. Reich and W. Reich, "A radio continuum survey of the northern sky at 1420 MHz-Part II,” Astron. Astrophys., Suppl. Ser., vol. 63, pp. 205-292, Jul. 1986.

[7] P. Reich, J. C. Testori, and W. Reich, "A radio continuum survey of the southern sky at $1420 \mathrm{MHz}$, the atlas of contour maps," Astron. Astrophys., vol. 376, no. 3, pp. 861-877, Sep. 2001.

[8] J. C. Testori, P. Reich, J. A. Bava, F. R. Colomb, E. E. Hurrel, J. J. Larrarte, W. Reich, and A. J. Sanz, "A radio continuum survey of the southern sky at $1420 \mathrm{MHz}$. Observations and data reduction," Astron. Astrophys., vol. 368, no. 3, pp. 1123-1132, Mar. 2001.

[9] P. M. W. Kalberla, W. B. Burton, D. Hartmann, E. M. Arnal, E. Bajaja, R. Morras, and W. G. L. Pöppel, "The Leiden/Argentine/Bonn (LAB) survey of galactic HI. Final data release of the combined LDS and IAR surveys with improved stray-radiation corrections," Astron. Astrophys., vol. 440, no. 2, pp. 775-782, Sep. 2005.

0] D. Hartmann and W. B. Burton, Atlas of Galactic Neutral Hydrogen. Cambridge, U.K.: Cambridge Univ. Press, 1997.

11] E. M. Arnal, E. Bajaja, J. J. Larrarte, R. Morras, and W. G. L. Pöppel, "A high sensitivity HI survey of the sky at $\delta \leq-25^{\circ}$," Astron. Astrophys. Suppl. Ser., vol. 142, pp. 35-40, Feb. 2000.

2] E. Bajaja, E. M. Arnal, J. J. Larrarte, R. Morras, W. G. L. Pöppel, and P. M. W. Kalberla, "A high sensitivity HI survey of the sky at $\delta \leq-25^{\circ}$. Final data release," Astron. Astrophys., vol. 440, pp. 767-773, Sep. 2005.

13] A. Wright and R. Otrupcek, Parkes Catalog. Epping, Australia: Australia Telescope Nat. Facility, 1990.

4] J. J. Condon, W. D. Cotton, E. W. Greisen, Q. F. Yin, R. A. Perley, G. B. Taylor, and J. J. Broderick, "The NRAO VLA sky survey," Astron. J., vol. 115, no. 5, pp. 1693-1716, May 1998.

5] P. Beckmann and A. Spizzichino, The Scattering of Electromagnetic Waves From Rough Surfaces. New York: Macmillan, 1963.

[16] A. G. Voronovich, "Small-slope approximation in wave scattering by rough surfaces," Sov. Phys._JETP, vol. 62, pp. 65-70, 1985.

17] A. Guissard, "Mueller and Kennaugh matrices in radar polarimetry," IEEE Trans. Geosci. Remote Sens., vol. 32, no. 3, pp. 590-597, May 1994.

8] C. Mätzler and P. W. Rosenkranz, "Dependence of microwave brightness temperature on bistatic surface scattering: Model functions and application to AMSU-A," IEEE Trans. Geosci. Remote Sens., vol. 45, no. 7, pp. 2130-2138, Jul. 2007.

9] D. S. S. L., Computation of the synthetic antenna directional gain as interface to L2 processor, synthetic antenna, Apr. 2005, Lisboa, Portugal: DEIMOS Space S.L. Tech. Note.

20] P. Waldteufel and S. Zine, "Approximating the weighting function to be used in the SMOS Level 2 processor," CBSA, Toulouse, France, 2005. Tech. Rep., 32 p..

1] J. Tournadre, B. Chapron, N. Reul, and D. C. Vandemark, "A satellite altimeter model for ocean slick detection," J. Geophys. Res., vol. 111 no. C4, p. C04 004, 2006.

2] V. N. Kudryavtsev, V. K. Makin, and B. Chapron, "Coupled sea surfaceatmosphere model 2. Spectrum of short wind waves," J. Geophys. Res., vol. 104, no. C4, pp. 7625-7639, 1999.

[23] M. Wolleben, T. L. Landecker, W. Reich, and R. Wielebinski, An Absolutely Calibrated Survey of Polarized Emission From the Northern Sky at $1.4 \mathrm{GHz}$, 2005. [Online]. Available: http://www.citebase.org/abstract? $\mathrm{id}=$ oai:arXiv.org:astro-ph/0510456

24] M. Wolleben, T. L. Landecker, W. Reich, and R. Wielebinski, "An absolutely calibrated survey of polarized emission from the northern sky at $1.4 \mathrm{GHz}$-Observations and data reduction," Astron. Astrophys., vol. 448, no. 1, pp. 411-424, Mar. 2006.

25] E. Carretti, S. Poppi, W. Reich, P. Reich, E. Fuerst, G. Bernardi, S. Cortiglioni and C. Sbarra, "Deep 1.4-GHz observations of diffuse polarized emission," Mon. Not. R. Astron. Soc., vol. 367, no. 1, pp 132-138, Mar. 2006. [Online] Available: http://www.citebase.org/abstract?id=oai: arXiv.org:astro-ph/0512286

[26] M. Haverkorn, B. M. Gaensler, N. M. McClure-Griffiths, J. M. Dickey, and A. J. Green, The Southern Galactic Plane Survey: Polarized Radio Continuum Observations and Analysis, 2006. [Online]. Available: http://www.citebase.org/abstract?id=oai:arXiv.org:astro-ph/0609010

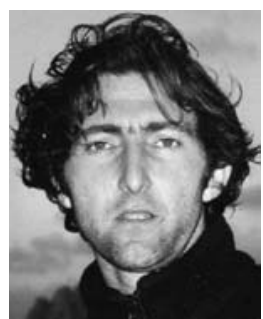

Nicolas Reul received the B.S. degree in marine 1042 science engineering from Toulon University, Toulon, 1043 France, in 1993 and the Ph.D. degree in physics (fluid 1044 mechanics) from the University of Aix-Marseille II, 1045 Marseille, France, in 1998.

1046

From 1999 to 2001, he was with the Depart- 1047 ment of Applied Marine Physics, Rosenstiel School 1048 of Marine and Atmospheric Science, University of 1049 Miami, Miami, FL, as a Postdoctoral Researcher in 1050 the team of Prof. M. Donelan. Since 2001, he has 1051 been a Research Scientist with the Spatial Oceanog- 1052 raphy Group, Laboratoire d'Océanographie Spatiale, Institut Français de 1053 Recherche et d'Exploitation de la Mer, Plouzané, France, where is responsible 1054 for activities concerning the SMOS satellite mission. The focus of his research 1055 program is the improvement of the understanding of the physical processes in 1056 the air-sea interface and passive/active remote sensing of the ocean surface. 1057 He has experience in applied mathematics, physical oceanography, and elec- 1058 tromagnetic wave theory and its application to ocean remote sensing. He is a 1059 member of the European Space Agency/SMOS Science Advisory Group. 1060

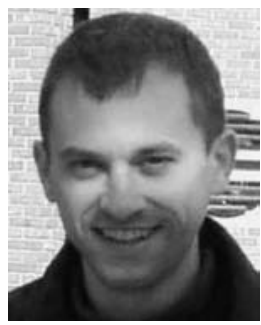

Joseph Tenerelli received the B.S. degree in 1061 atmospheric sciences from the University of 1062 Washington, Seattle, in 1994.

From 1999 to 2005, he was a Research Asso- 1064 ciate with the Rosenstiel School of Marine and At- 1065 mospheric Science, University of Miami, Miami, FL, 1066 where he was part of a team that developed a coupled 1067 atmosphere-ocean-surface wave model with vortex- 1068 following mesh refinement suitable for simulating 1069 hurricanes. Since April 2005, he has been a Research 1070 Engineer with the Laboratoire d'Océanographie 1071 Spatiale, Institut Français de Recherche et d'Exploitation de la Mer, Plouzané, 1072 France, as part of a team that is developing an algorithm for retrieving sea 1073 surface salinity from L-band radiometric measurements (the European Space 1074 Agency's Soil Moisture and Ocean Salinity project).

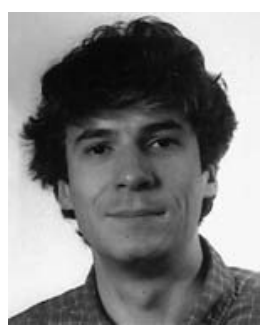

Nicolas Floury received the Engineering degree in 1076 telecommunication techniques from the Ecole Na- 1077 tionale Supérieure des Télécommunications, Paris, 1078 France, in 1993 and the Ph.D. degree in physics ap- 1079 plied to remote sensing from the Université Paris 7, 1080 Paris, in 1999.

Since 1999, he has been with the Wave Interaction 1082 and Propagation Group, Electromagnetics and Space 1083 Environments Division, European Space Research 1084 and Technology Centre (ESTEC), European Space 1085 Agency, Noordwijk, The Netherlands. His interests 1086 are in electromagnetic modeling and signal processing applied to the study of 1087 the interaction between microwaves and natural media.

1088

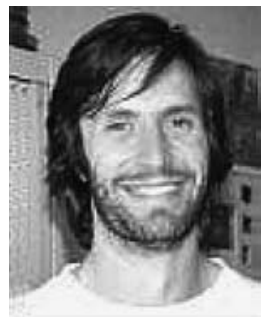

Bertrand Chapron received the B.Eng. degree from 1089 the Institut National Polytechnique de Grenoble, 1090 Grenoble, France, in 1984 and the Ph.D. degree in 1091 physics (fluid mechanics) from the University of 1092 Aix-Marseille II, Marseille, France, in 1988.

He was with the NASA Goddard Space Flight 1094 Center Wallops Flight Facility, Wallops Island, VA, 1095 for three years as a Postdoctoral Research Asso- 1096 ciate. He is currently a Research Scientist and the 1097 Head of the Spatial Oceanography Group, Labora- 1098 toire d'Océanographie Spatiale, Institut Français de 1099 Recherche et d'Exploitation de la Mer, Plouzané, France, where he is responsi- 1100 ble for the Centre ERS Archivage et Traitement. He has been a Coinvestigator 1101 and Principal Investigator in several projects of the European Space Agency 1102 (e.g., ENVISAT and Global Navigation Satellite System), NASA, and Centre 1103 National d'Études Spatiales (e.g., TOPEX/POSEIDON and JASON). He was 1104 also responsible (with H. Johnsen of NORUT) for the ENVISAT ASAR-wave 1105 mode algorithms and scientific preparation for the ENVISAT wind and wave 1106 products. He has experience in applied mathematics, physical oceanography, 1107 and electromagnetic wave theory and its application to ocean remote sensing. 1108 